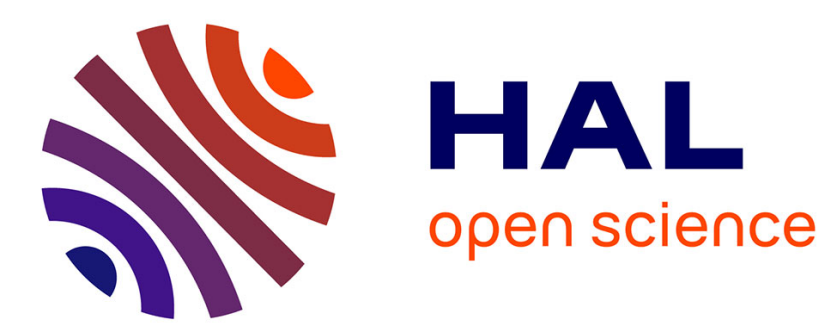

\title{
Boundary controllability for finite-differences semi-discretizations of a clamped beam equation
}

Nicolae Cîndea, Sorin Micu, Ionel Roventa

\section{To cite this version:}

Nicolae Cîndea, Sorin Micu, Ionel Roventa. Boundary controllability for finite-differences semidiscretizations of a clamped beam equation. 2016. hal-01314808

\section{HAL Id: hal-01314808 \\ https://hal.science/hal-01314808}

Preprint submitted on 12 May 2016

HAL is a multi-disciplinary open access archive for the deposit and dissemination of scientific research documents, whether they are published or not. The documents may come from teaching and research institutions in France or abroad, or from public or private research centers.
L'archive ouverte pluridisciplinaire HAL, est destinée au dépôt et à la diffusion de documents scientifiques de niveau recherche, publiés ou non, émanant des établissements d'enseignement et de recherche français ou étrangers, des laboratoires publics ou privés. 


\title{
Boundary controllability for finite-differences semi-discretizations of a clamped beam equation
}

\author{
Nicolae Cîndea \\ Laboratoire de Mathématiques, Université Blaise Pascal Clermont-Ferrand \\ Campus des Cézeaux - 3 place Vasarely, 63178 Aubière, France \\ Nicolae.Cindea@math. univ-bpclermont.fr \\ Sorin Micu \\ Department of Mathematics, University of Craiova \\ Craiova, 200585, Romania \\ sd_micu@yahoo.com \\ Ionel Rovenţa \\ Department of Mathematics, University of Craiova \\ Craiova, 200585, Romania \\ ionelroventa@yahoo.com
}

May 11, 2016

\begin{abstract}
This article deals with the boundary observability properties of a space finite-differences semidiscretization of the clamped beam equation. We make a detailed spectral analysis of the system and, by combining numerical estimates with asymptotic expansions, we localize all the eigenvalues of the corresponding discrete operator depending on the mesh size $h$. Then, an Ingham's type inequality and a discrete multiplier method allow us to deduce that the uniform (with respect to $h$ ) observability property holds if and only if the eigenfrequencies are filtered out in the range $O\left(1 / h^{4}\right)$.
\end{abstract}

\section{Introduction}

We consider the following equation modelling the boundary controlled vibration of an elastic beam clamped at both extremities

$$
\begin{cases}\ddot{u}(x, t)+\partial_{x}^{4} u(x, t)=0, & (x, t) \in(0,1) \times(0, T) \\ u(0, t)=u(1, t)=0, & t \in(0, T) \\ \partial_{x} u(0, t)=0, \quad \partial_{x} u(1, t)=v(t), & t \in(0, T) \\ u(x, 0)=u_{0}(x), \quad \dot{u}(x, 0)=u_{1}(x), & x \in(0,1) .\end{cases}
$$

In (1.1) $u=u(x, t)$ denotes the position of the beam at time $t \geqslant 0$ and point $x \in[0,1]$ and $v=v(t)$ represents the boundary control acting on the extremity $x=1$. Moreover, $\dot{u}$ denotes the derivative of $u$ with respect to time. The controllability problem associated to (1.1) reads as follows: given $T>0$, for every initial data $\left(u_{0}, u_{1}\right) \in L^{2}(0,1) \times H^{-2}(0,1)$ there exists a control $v \in L^{2}(0, T)$ such that the solution of (1.1) verifies

$$
u(x, T)=\dot{u}(x, T)=0 \quad(x \in(0,1)) .
$$


It has been proved that, for every $T>0$, the above controllability problem has a positive answer. For details see [11, Section 6.10] for a solution using semigroups theory or [9] for a solution using multiplier method. Moreover, it is well known that this controllability property is equivalent with an observability property ensuring that there exists a constant $K>0$ such that the following inequality holds

$$
\left\|\left(y_{0}, y_{1}\right)\right\|_{H_{0}^{2}(0,1) \times L^{2}(0,1)}^{2} \leqslant K \int_{0}^{T}\left|\partial_{x}^{2} y(1, t)\right|^{2} d t \quad\left(\left(y_{0}, y_{1}\right) \in H_{0}^{2}(0,1) \times L^{2}(0,1)\right),
$$

where $(y, \dot{y})$ is the solution of the following adjoint homogeneous equation

$$
\begin{cases}\ddot{y}(x, t)+\partial_{x}^{4} y(x, t)=0, & (x, t) \in(0,1) \times(0, T) \\ y(0, t)=y(1, t)=0, & t \in(0, T) \\ \partial_{x} y(0, t)=\partial_{x} y(1, t)=0, & t \in(0, T) \\ y(x, 0)=y_{0}(x), \quad \dot{y}(x, 0)=y_{1}(x), & x \in(0,1)\end{cases}
$$

The main objective of this work is to study the controllability properties of a finite-differences semidiscrete approximation of (1.1). More precisely, let $N$ be the number of discretization points uniformly distributed in the interval $(0,1)$ and let $h=\frac{1}{N+1}$. The finite-differences semi-discretization of system (1.1) is the following:

$$
\begin{cases}\ddot{u}_{j}(t)+\frac{1}{h^{4}}\left(u_{j+2}(t)-4 u_{j+1}(t)+6 u_{j}(t)-4 u_{j-1}(t)+u_{j-2}(t)\right)=0, & 1 \leq j \leq N, t \in(0, T) \\ u_{0}(t)=0, \quad u_{N+1}(t)=0, & t \in(0, T) \\ u_{-1}(t)=u_{1}(t), \quad u_{N+2}(t)=u_{N}(t)+h v_{h}(t), & t \in(0, T) \\ u_{j}(0)=u_{j}^{0}, \quad \dot{u}_{j}(0)=u_{j}^{1}, & 1 \leq j \leq N,\end{cases}
$$

where for every $1 \leq j \leq N, u_{j}(t)$ approximates the solution $u(j h, t)$ of $(1.1)$ and $\left(u_{j}^{0}, u_{j}^{1}\right)$ is the approximation of the initial data of (1.1). In order to tackle the boundary conditions, two extra functions $u_{-1}(t)$ and $u_{N+2}(t)$ have been introduced. The controllability problem associated to (1.5) reads as follows: for a given $T>0$ and $\left(u_{j}^{0}, u_{j}^{1}\right)_{1 \leq j \leq N} \in \mathbb{C}^{2 N}$ there exists a control $v_{h} \in L^{2}(0, T)$ such that the solution of (1.5) satisfies:

$$
u_{j}(T)=\dot{u}_{j}(T)=0 \quad(1 \leq j \leq N) .
$$

We remark that system (1.5) can be equivalently written in the following vectorial form

$$
\left\{\begin{array}{l}
\ddot{U}_{h}(t)+A_{h} U_{h}(t)=F_{h}(t), \quad t \in(0, T) \\
U_{h}(0)=U_{h}^{0}, \quad \dot{U}_{h}(0)=U_{h}^{1}
\end{array}\right.
$$

where

$$
U_{h}^{0}=\left(\begin{array}{c}
u_{1}^{0} \\
u_{2}^{0} \\
\vdots \\
u_{N}^{0}
\end{array}\right), \quad U_{h}^{1}=\left(\begin{array}{c}
u_{1}^{1} \\
u_{2}^{1} \\
\vdots \\
u_{N}^{1}
\end{array}\right), \quad U_{h}(t)=\left(\begin{array}{c}
u_{1}(t) \\
u_{2}(t) \\
\vdots \\
u_{N}(t)
\end{array}\right), \quad F_{h}(t)=-\frac{1}{h^{3}}\left(\begin{array}{c}
0 \\
0 \\
\vdots \\
v_{h}
\end{array}\right)
$$

and $A_{h}=\frac{1}{h^{4}} A$, with $A$ the matrix in $\mathcal{M}_{N \times N}(\mathbb{C})$ defined by

$$
A=\left(\begin{array}{rrrrrrrrr}
7 & -4 & 1 & 0 & \ldots & \ldots & \ldots & \ldots & 0 \\
-4 & 6 & -4 & 1 & 0 & \ldots & \ldots & \ldots & 0 \\
1 & -4 & 6 & -4 & 1 & 0 & \ldots & \ldots & 0 \\
0 & 1 & -4 & 6 & -4 & 1 & 0 & \ldots & 0 \\
\vdots & \ddots & \ddots & \ddots & \ddots & \ddots & \ddots & \ddots & \vdots \\
0 & \ldots & 0 & 1 & -4 & 6 & -4 & 1 & 0 \\
0 & \ldots & \ldots & 0 & 1 & -4 & 6 & -4 & 1 \\
0 & \ldots & \ldots & \ldots & 0 & 1 & -4 & 6 & -4 \\
0 & \ldots & \ldots & \ldots & \ldots & 0 & 1 & -4 & 7
\end{array}\right)
$$


Let us define in $\mathbb{C}^{N}$ the canonical inner product given by

$$
\langle F, G\rangle_{0}=h \sum_{i=1}^{N} f_{i} \bar{g}_{i} \quad\left(F=\left(f_{i}\right)_{1 \leqslant i \leqslant N}, G=\left(g_{i}\right)_{1 \leqslant i \leqslant N} \in \mathbb{C}^{N}\right),
$$

and let $\|\cdot\|_{0}$ be its corresponding norm. In $\mathbb{C}^{N}$, we also introduce the inner product

$$
\langle F, G\rangle_{2}=\left\langle A_{h} F, G\right\rangle_{0} \quad\left(F, G \in \mathbb{C}^{N}\right),
$$

with the corresponding norm denoted by $\|\cdot\|_{2}$. We remark that, whereas the norm $\|\cdot\|_{0}$ is a discrete version of the norm in $L^{2}(0,1)$, the norm $\|\cdot\|_{2}$ discretizes the usual norm in $H_{0}^{2}(0,1)$. Finally, let us introduce the inner product in $\mathbb{C}^{2 N}$ defined by

$$
\left\langle\left(\begin{array}{l}
F^{1} \\
F^{2}
\end{array}\right),\left(\begin{array}{l}
G^{1} \\
G^{2}
\end{array}\right)\right\rangle=\left\langle F^{1}, G^{1}\right\rangle_{2}+\left\langle F^{2}, G^{2}\right\rangle_{0} \quad\left(\left(\begin{array}{l}
F^{1} \\
F^{2}
\end{array}\right),\left(\begin{array}{l}
G^{1} \\
G^{2}
\end{array}\right) \in \mathbb{C}^{2 N}\right)
$$

with the corresponding norm denoted $\|\cdot\|$.

One expects that the family of discrete controls $\left(v_{h}\right)_{h>0}$ for (1.5) approximates a control $v$ of the continuous equation (1.1) and converges to it as $h$ tends to zero. The fact that this is not generally true has been proved in several semi-discrete settings corresponding to the linear wave equation [7] or hinged beam equation [10]. The non convergence result is due to the bad numerical approximation of the highest eigenvalues and eigenvectors, which do not reflect anymore the properties of their continuous counterparts. This phenomenon can be also seen at the level of the discrete observability inequality corresponding to the problem. Indeed, if the observability constant is not uniformly bounded with respect to the mesh size $h$, then it can be proved that there exist initial data for which the family of minimal norm discrete controls diverges when $h$ tends to zero. Reciprocally, if the observability constant is uniformly bounded in $h$, the convergence of the discrete controls is ensured. In fact, the study of the corresponding discrete observability inequality shows not only the convergence or not of the numerical scheme, but also indicates possible cures of the defects. This is the case for the hinged beam equation which was considered in [10]. More precisely, from the spectral analysis of the problem and the study of the corresponding observability inequality, two possibilities to ensure the convergence of the scheme have been proposed in [10]: by filtering out the spurious high frequencies or by adding an extra boundary control. An alternative method to ensure the uniform controllability result consists in adding a vanishing numerical viscosity and has been analyzed in [1].

The aim of this paper is to study the discrete observability property corresponding to the controlled problem (1.5) which reads as follows: there exists a constant $K_{h}$ such that the following inequality holds

$$
\left\|Y_{h}^{0}\right\|_{2}^{2}+\left\|Y_{h}^{1}\right\|_{0}^{2} \leqslant K_{h} \int_{0}^{T}\left|\frac{Y_{h N}(t)}{h^{2}}\right|^{2} d t
$$

for any $\left(\begin{array}{c}Y_{h}^{0} \\ Y_{h}^{1}\end{array}\right) \in \mathbb{C}^{2 N}$, where $\left(\begin{array}{c}Y_{h} \\ \dot{Y}_{h}\end{array}\right)$ is the solution of the discrete version of (1.4) given by

$$
\left\{\begin{array}{l}
\ddot{Y}_{h}(t)+A_{h} Y_{h}(t)=0, \quad t \in(0, T) \\
Y_{h}(0)=Y_{h}^{0}, \quad \dot{Y}_{h}(0)=Y_{h}^{1}
\end{array}\right.
$$

We remak that (1.11) is a discrete version of (1.3), since the norms $\|\cdot\|_{0}$ and $\|\cdot\|_{2}$ stand for approximations of the norms in $L^{2}(0,1)$ and $H_{0}^{2}(0,1)$, respectively. Moreover, by taking into account the boundary relations $Y_{h N+1}=0$ and $Y_{h N+2}=Y_{h N}$, we deduce that $\frac{2 Y_{h N}(t)}{h^{2}}$ is an approximation of $\partial_{x}^{2} y(1, t)$. If $K_{h}$ does not depend of $h$, we say that (1.6) is uniformly observable. The study of the behavior of the constant $K_{h}$ in (1.11) with respect to $h$ is the main objective of this paper. The uniform boundedness of $K_{h}$ with respect to $h$ would ensure directly the convergence of the controls $\left(v_{h}\right)_{h>0}$ for (1.5) to a control $v$ of (1.1). On the contrary, if $K_{h}$ tends to infinity as $h$ goes to zero, some kind of filtration is needed to ensure the convergence.

In the examples of the linear wave and hinged beam equations mentioned before the observability constant is not uniformly bounded with respect to the discretization parameter $h$. Our main result states 
that, for the clamped beam equation (1.4), the uniform observability inequality associated to (1.12) does not hold either. Hence, $K_{h}$ tends to infinity as $h$ goes to zero. Nevertheless, by filtering out the high frequencies in the range $O\left(\frac{1}{h^{4}}\right)$, the uniform observability inequality is restored.

These results are similar to the ones obtained for the hinged beam equation. The main difference is that, for the clamped beam (1.4), the corresponding discrete finite-differences operator $A_{h}$ is no more the square of finite-differences discrete Laplacian and the eigenvalues cannot be explicitly computed. This makes the Fourier analysis of the solutions of (1.12), and consequently the proof of the results concerning the observability inequality (1.11), more intricate. Using some algebraic computations combined with asymptotic estimates and Rouché's theorem, we are able to localize the eigenvalues of the discrete operator $A_{h}$ driving system (1.6). Moreover, we also obtain estimates for the eigenvectors of $A_{h}$ by using asymptotic techniques and a discrete multiplier method.

Finally, let us remark that our analysis shows precisely how and when the observability inequality begins to deteriorate. It gives more precise results than the general ones obtained for the abstract hyperbolic equations deduced in $[3,4]$. For instance, we are able to deduce that the optimal filtering range ensuring the uniform observability is $O\left(\frac{1}{h^{4}}\right)$ and that the main defect comes from an error of order of $h^{-1}$ in the approximation of the second derivative $\partial_{x}^{2} u(1, t)$ affecting the highest frequencies in that range.

The remaining part of this paper is organized as follows. In Section 2 we analyze the eigenvalues and eigenvectors of the discrete operator by dividing the spectrum in two parts: the low and the high one. On the one side, the low frequencies are tackled by using a classical numerical convergence result, and, on the other side, the high frequencies are studied by using an argument based on Rouché's Theorem. In the same Section 2 we give some lower estimates for the last component of the eigenvectors again by analyzing separately the cases of the high and the low frequencies. Section 3 formulates and proves the main results of our paper concerning the observability inequality (1.11). Finally, in Section 4, we numerically illustrate the obtained results by considering some numerical experiments for the approximation of the controls.

\section{Spectral properties of the matrix $A$}

The main objective of this paper is to study the inequality (1.11) by using a spectral decomposition of the operator $A_{h}$ and a Fourier expansion of solutions $\left(\begin{array}{c}Y_{h} \\ \dot{Y}_{h}\end{array}\right)$ of (1.12). The aim of this section is to offer the needed spectral information concerning the discrete operator $A_{h}$. In a first step we characterize the eigenvalues and eigenvectors of the matrix $A$ as solutions of some trigonometric equations. After that, we obtain estimates for the high part of the spectrum of $A$ by using the Rouchés theorem and asymptotic expansions. The low part of the spectrum is analyzed with the aid of a numerical convergence result and a discrete multiplier method.

\subsection{Algebraic properties}

Firstly, we have the following simple result which is a consequence of the elementary properties of the matrix $A$.

Proposition 2.1. The matrix A given by (1.7) has only real eigenvalues $\left(\lambda_{n}\right)_{1 \leqslant n \leqslant N} \subset(0,16)$ and there exists an orthonormal basis in $\mathbb{C}^{N}$ (with respect to the canonical inner product (1.8)) consisting of eigenvectors $\left(\phi^{n}\right)_{1 \leqslant n \leqslant N}$ of $A$.

Proof. We remark that $A$ is a symmetric matrix and it can be written as $A=B^{2}+D$, where

$$
B=\left(\begin{array}{rrrrrrr}
2 & -1 & 0 & \ldots & \ldots & \ldots & 0 \\
-1 & 2 & -1 & 0 & \ldots & \ldots & 0 \\
0 & -1 & 2 & -1 & 0 & \ldots & 0 \\
\vdots & \ddots & \ddots & \ddots & \ddots & \ddots & \vdots \\
0 & \ldots & 0 & -1 & 2 & -1 & 0 \\
0 & \ldots & \ldots & 0 & -1 & 2 & -1 \\
0 & \ldots & \ldots & \ldots & 0 & -1 & 2
\end{array}\right), \quad D=\left(\begin{array}{rrrrrrr}
2 & 0 & 0 & \ldots & \ldots & \ldots & 0 \\
0 & 0 & 0 & 0 & \ldots & \ldots & 0 \\
0 & 0 & 0 & 0 & 0 & \ldots & 0 \\
\vdots & \ddots & \ddots & \ddots & \ddots & \ddots & \vdots \\
0 & \ldots & 0 & 0 & 0 & 0 & 0 \\
0 & \ldots & \ldots & 0 & 0 & 0 & 0 \\
0 & \ldots & \ldots & \ldots & 0 & 0 & 2
\end{array}\right) .
$$


Since $B$ is positive defined and $D$ is positive semi-defined it follows that $A$ is positive definite. Hence, the eigenvalues of $A$ are real positive numbers. To find an upper bound for the eigenvalues we use the well known result of Gershgorin (see, for instance, [5, Corollary 6.1.5, p. 346]) and deduce that every eigenvalue of $A$ verifies

$$
\left|\lambda_{n}\right| \leqslant \max _{i \in\{1,2, \ldots, N\}} \sum_{j=1}^{N}\left|a_{i j}\right|=16 \quad(1 \leq n \leq N) .
$$

To show that a strict inequality takes place in the above estimate it is sufficient to remark that the matrix $A$ has the property $S C$ and that the point of coordinates $(16,0)$ is not on the boundaries of all Gershgorin discs (see [5, Better Theorem 6.2.8, p. 356]). The remaining part of the proof follows from standard results for symmetric matrices.

The following proposition gives an algebraic characterization of the eigenvalues and eigenvectors of the matrix $A$.

Proposition 2.2. With the above notation, $\lambda$ is a eigenvalue of the matrix $A$ if and only if verifies one of the following relations

$$
\cos \left((N+1) \arg \left(X_{4}\right)\right)=\frac{8 X_{1}^{N+1}-\sqrt{\lambda} X_{1}^{2(N+1)}-\sqrt{\lambda}}{2\left(2 X_{1}^{2(N+1)}-\sqrt{\lambda} X_{1}^{N+1}+2\right)}, \quad \sin \left((N+1) \arg \left(X_{4}\right)\right)>0,
$$

or

$$
\cos \left((N+1) \arg \left(X_{4}\right)\right)=\frac{8 X_{1}^{N+1}+\sqrt{\lambda} X_{1}^{2(N+1)}+\sqrt{\lambda}}{2\left(2 X_{1}^{2(N+1)}+\sqrt{\lambda} X_{1}^{N+1}+2\right)}, \quad \sin \left((N+1) \arg \left(X_{4}\right)\right)<0
$$

where for each $j \in\{1,2,3,4\}$ the numbers $X_{j}$ are given by

$$
\begin{aligned}
& X_{1,2}=\frac{2+\sqrt{\lambda} \pm \sqrt{(2+\sqrt{\lambda})^{2}-4}}{2}, \\
& X_{3,4}=\frac{2-\sqrt{\lambda} \pm i \sqrt{4-(2-\sqrt{\lambda})^{2}}}{2} .
\end{aligned}
$$

Moreover, to each eigenvalue $\lambda$ of $A$ corresponds a unitary eigenvector $\phi=\left(\phi_{1}, \phi_{2}, \ldots, \phi_{N}\right)^{\prime}$ given by

$$
\phi_{k}=C_{1} X_{1}^{k}+C_{2} X_{2}^{k}+C_{3} X_{3}^{k}+C_{4} X_{4}^{k} \quad(k \in\{1,2, \ldots, N\})
$$

where

$$
\begin{gathered}
C_{1}=\frac{\mathfrak{C}}{X_{1}^{N+1} r_{N}^{1}}, \\
C_{2}=-\frac{\mathfrak{C}}{X_{2}^{N+1} r_{N}^{2}}, \\
C_{3}=-\alpha C_{1}\left(\frac{X_{1}}{X_{3}}\right)^{N+1}-\beta C_{2}\left(\frac{X_{2}}{X_{3}}\right)^{N+1}, \\
C_{4}=-\beta C_{1}\left(\frac{X_{1}}{X_{4}}\right)^{N+1}-\alpha C_{2}\left(\frac{X_{2}}{X_{4}}\right)^{N+1}, \\
r_{N}^{1}=\sqrt{\left(\left(\frac{X_{4}}{X_{1}}\right)^{N+1}-1\right)\left(\left(\frac{X_{3}}{X_{1}}\right)^{N+1}-1\right)}, \\
r_{N}^{2}=\sqrt{\left(\left(\frac{X_{4}}{X_{2}}\right)^{N+1}-1\right)\left(\left(\frac{X_{3}}{X_{2}}\right)^{N+1}-1\right)},
\end{gathered}
$$




$$
\begin{gathered}
\alpha=\frac{1}{2}\left(1-i \frac{R_{+}}{R_{-}}\right), \quad \beta=\frac{1}{2}\left(1+i \frac{R_{+}}{R_{-}}\right), \\
R_{+}=\sqrt{(2+\sqrt{\lambda})^{2}-4}, \quad R_{-}=\sqrt{4-(2-\sqrt{\lambda})^{2}} .
\end{gathered}
$$

The positive constant $\mathfrak{C}$ appearing in (2.6)-(2.7) is chosen such that $\|\phi\|_{0}=1$.

Proof. Let $\lambda$ be an eigenvalue of the matrix $A$ and $\phi$ a corresponding eigenvector. Note that, by taking $\phi_{0}=\phi_{N+1}=0, \phi_{-1}=\phi_{1}$ and $\phi_{N+2}=\phi_{N}$, we deduce from the equality $A \phi=\lambda \phi$ that

$$
\phi_{k-2}-4 \phi_{k-1}+(6-\lambda) \phi_{k}-4 \phi_{k+1}+\phi_{k+2}=0 \quad(1 \leqslant k \leqslant N)
$$

Equality (2.14) represents a recurrence relation of order 4 and its characteristic equation is given by

$$
x^{4}-4 x^{3}+(6-\lambda) x^{2}-4 x+1=0 .
$$

Since $x \neq 0$, dividing $(2.15)$ by $x^{2}$, we obtain:

$$
\left(x+\frac{1}{x}\right)^{2}-4\left(x+\frac{1}{x}\right)+4-\lambda=0
$$

and, denoting $s=x+\frac{1}{x}$, we obtain the following second order equation $s^{2}-4 s+4-\lambda=0$ with the solutions $s_{1,2}=2 \pm \sqrt{\lambda}$. Therefore, going back to the variable $x$ and taking into account that $\lambda \in(0,16)$, we obtain that equation (2.15) has four different solutions $X_{j}(j \in\{1,2,3,4\})$ given by (2.3)-(2.4). From classical results about recursive sequences there exist constants $C_{1}, C_{2}, C_{3}, C_{4} \in \mathbb{C}$ such that (2.5) holds.

From the boundary conditions $\phi_{0}=\phi_{N+1}=0, \phi_{-1}=\phi_{1}$ and $\phi_{N+2}=\phi_{N}$, we deduce that the constants $C_{j}, j \in\{1,2,3,4\}$, satisfy the following system:

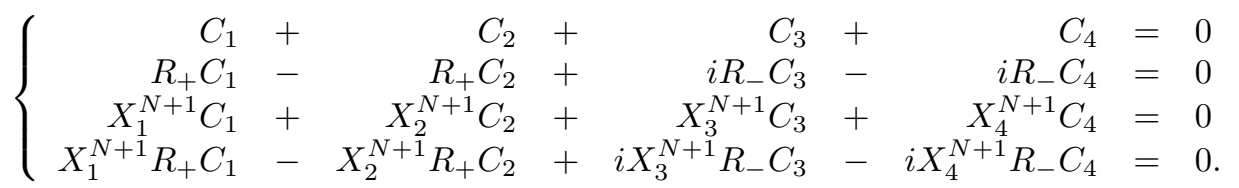

From the first two equations in system (2.16) we extract

$$
\begin{aligned}
& C_{3}=-\frac{1}{2}\left(1-i \frac{R_{+}}{R_{-}}\right) C_{1}-\frac{1}{2}\left(1+i \frac{R_{+}}{R_{-}}\right) C_{2}, \\
& C_{4}=-\frac{1}{2}\left(1+i \frac{R_{+}}{R_{-}}\right) C_{1}-\frac{1}{2}\left(1-i \frac{R_{+}}{R_{-}}\right) C_{2},
\end{aligned}
$$

and from the last two equations

$$
\begin{aligned}
& C_{3}=-\frac{1}{2}\left(1-i \frac{R_{+}}{R_{-}}\right) \frac{X_{1}^{N+1}}{X_{3}^{N+1}} C_{1}-\frac{1}{2}\left(1+i \frac{R_{+}}{R_{-}}\right) \frac{X_{2}^{N+1}}{X_{3}^{N+1}} C_{2}, \\
& C_{4}=-\frac{1}{2}\left(1+i \frac{R_{+}}{R_{-}}\right) \frac{X_{1}^{N+1}}{X_{4}^{N+1}} C_{1}-\frac{1}{2}\left(1-i \frac{R_{+}}{R_{-}}\right) \frac{X_{2}^{N+1}}{X_{4}^{N+1}} C_{2},
\end{aligned}
$$

thus (2.8-2.9) hold. Putting together these relations, we obtain the following system verified by $C_{1}$ and $C_{2}$ :

$$
\left\{\begin{array}{l}
\left(1-i \frac{R_{+}}{R_{-}}\right)\left(1-\frac{X_{1}^{N+1}}{X_{3}^{N+1}}\right) C_{1}+\left(1+i \frac{R_{+}}{R_{-}}\right)\left(1-\frac{X_{2}^{N+1}}{X_{3}^{N+1}}\right) C_{2}=0 \\
\left(1+i \frac{R_{+}}{R_{-}}\right)\left(1-\frac{X_{1}^{N+1}}{X_{4}^{N+1}}\right) C_{1}+\left(1-i \frac{R_{+}}{R_{-}}\right)\left(1-\frac{X_{2}^{N+1}}{X_{4}^{N+1}}\right) C_{2}=0 .
\end{array}\right.
$$

Since we are not interested to the null solution to (2.17) (and, respectively, to (2.16)), the determinant associated to system (2.17) should be equal to zero, i.e.,

$$
\left(1-i \frac{R_{+}}{R_{-}}\right)^{2}\left(X_{3}^{N+1}-X_{1}^{N+1}\right)\left(X_{4}^{N+1}-X_{2}^{N+1}\right)=\left(1+i \frac{R_{+}}{R_{-}}\right)^{2}\left(X_{4}^{N+1}-X_{1}^{N+1}\right)\left(X_{3}^{N+1}-X_{2}^{N+1}\right)
$$


or, in a slightly different but equivalent form,

$$
\left(\frac{R_{-}-i R_{+}}{R_{-}+i R_{+}}\right)^{2}=\frac{\left(X_{4}^{N+1}-X_{1}^{N+1}\right)\left(X_{3}^{N+1}-X_{2}^{N+1}\right)}{\left(X_{3}^{N+1}-X_{1}^{N+1}\right)\left(X_{4}^{N+1}-X_{2}^{N+1}\right)}
$$

Moreover, we easily remark that

$$
\frac{R_{-}-i R_{+}}{R_{-}+i R_{+}}=-\frac{1}{4}(\sqrt{\lambda}+i \sqrt{16-\lambda}) .
$$

By choosing $C_{1}$ as in (2.6) then from (2.17) we obtain $C_{2}$ as in (2.7).

Let $\arg \left(X_{4}\right) \in[0,2 \pi)$ be the argument of the complex number $X_{4}$. It is easy to see from (2.3)-(2.4) that $X_{2}=\frac{1}{X_{1}}$ and $X_{3}=\bar{X}_{4}$. Using this notation and the trigonometric form of $X_{4}$, we obtain from (2.18) that $\lambda$ is an eigenvalue of the matrix $A$ if and only if it verifies

$$
\frac{\sqrt{\lambda}}{4}=\frac{2-\left(X_{1}^{N+1}+\frac{1}{X_{1}^{N+1}}\right) \cos \left((N+1) \arg \left(X_{4}\right)\right)}{X_{1}^{N+1}+\frac{1}{X_{1}^{N+1}}-2 \cos \left((N+1) \arg \left(X_{4}\right)\right)}, \quad \sin \left((N+1) \arg \left(X_{4}\right)\right)>0
$$

or

$$
-\frac{\sqrt{\lambda}}{4}=\frac{2-\left(X_{1}^{N+1}+\frac{1}{X_{1}^{N+1}}\right) \cos \left((N+1) \arg \left(X_{4}\right)\right)}{X_{1}^{N+1}+\frac{1}{X_{1}^{N+1}}-2 \cos \left((N+1) \arg \left(X_{4}\right)\right)}, \quad \sin \left((N+1) \arg \left(X_{4}\right)\right)<0 .
$$

This is equivalent to (2.1)-(2.2) and the proof of the proposition is complete.

\subsection{Spectral properties of the high frequencies}

In this section we asymptotically localize the largest eigenvalues of the matrix $A$ which, according to Proposition 2.2, verify equations (2.1) or (2.2). In order to do this, we need to rewrite the equations in a more tractable form. Firstly, let us remark that any number $\lambda \in(0,16)$ may be written as

$$
\lambda=16 \sin ^{4}\left(\frac{h z}{2}\right)
$$

for some $z \in\left(0, \frac{\pi}{h}\right)$. With this notation, from (2.3) and (2.4) we obtain that $\arg \left(X_{4}\right)=2 \pi-z h$ and

$$
\frac{8 X_{1}^{N+1} \pm \sqrt{\lambda} X_{1}^{2(N+1)} \pm \sqrt{\lambda}}{2\left(2 X_{1}^{2(N+1)} \pm \sqrt{\lambda} X_{1}^{N+1}+2\right)}= \pm \sin ^{2}\left(\frac{h z}{2}\right)+\frac{2\left(1-\sin ^{4}\left(\frac{h z}{2}\right)\right) r^{N+1}(z)}{r^{2(N+1)}(z) \pm 2 \sin ^{2}\left(\frac{h z}{2}\right) r^{N+1}(z)+1} .
$$

Let us define the complex functions

$$
g^{ \pm}(z)=\cos z \pm \sin ^{2}\left(\frac{z h}{2}\right) \text { and } f^{ \pm}(z)=g^{ \pm}(z)-\frac{2\left(1-\sin ^{4}\left(\frac{h z}{2}\right)\right) r^{N+1}(z)}{r^{2(N+1)}(z) \mp 2 \sin ^{2}\left(\frac{h z}{2}\right) r^{N+1}(z)+1},
$$

where $r(z)$ is a notation for $X_{1}$, namely

$$
r(z)=1+2 \sin ^{2}\left(\frac{z h}{2}\right)+2 \sqrt{\sin ^{2}\left(\frac{z h}{2}\right)\left(1+\sin ^{2}\left(\frac{z h}{2}\right)\right)} .
$$

Consequently, equations (2.1) and (2.2) are equivalent with $f^{+}(z)=0$ and $f^{-}(z)=0$, respectively. In the remaining part of this section, our aim is to get information about the zeros of the functions $f^{ \pm}$ by comparing them with the simpler functions $g^{ \pm}$and by using the Rouché's Theorem. 
Firstly, we have the following immediate result.

Lemma 2.3. Each equation $g^{ \pm}(z)=0$ has exactly one real zero $z_{n}^{ \pm}$in each interval $(n \pi,(n+1) \pi]$, for $0 \leqslant n \leqslant N$. Moreover, we have that

$$
\left\{\begin{array}{l}
z_{n}^{+} \in\left[n \pi, n \pi+\frac{\pi}{2}\right] \text { if } n \text { is odd } \\
z_{n}^{-} \in\left[n \pi, n \pi+\frac{\pi}{2}\right] \text { if } n \text { is even. }
\end{array}\right.
$$

Remark 2.4. As mentioned before, we are interested only in the values $z \in\left(0, \frac{\pi}{h}\right)$. The previous lemma ensures that each equation $g^{ \pm}(z)=0$ has exactly $N+1$ real zeros in the interval $\left(0, \frac{\pi}{h}\right)$. Since the first eigenvalue of the matrix $B$ defined in Proposition 2.1 is $\widetilde{\lambda}_{1}=4 \sin ^{2}\left(\frac{\pi h}{2}\right)$, it follows that the eigenvalues of our matrix $A$ are larger than $\left(\widetilde{\lambda}_{1}\right)^{2}$. Hence, if we are looking for eigenvalues $\lambda$ of the matrix $A$ in the form (2.21), then we should consider only values $z \geqslant \pi$.

For every $\delta>0$ and every $n \in\{1,2, \ldots, N\}$, we define the simple curves $\Gamma_{n}^{ \pm}(\delta)=\partial D_{n}^{ \pm}(\delta)$ where

$$
D_{n}^{ \pm}(\delta)=\left\{z \in \mathbb{C}|| z-z_{n}^{ \pm} \mid<\delta\right\}
$$

Moreover, for any $L \in\left(0, N+\frac{1}{2}\right)$, we consider the domain

$$
D(L)=\left\{z \in \mathbb{C}|| \Im(z) \mid \leqslant 1, L \pi \leqslant \Re(z) \leqslant N \pi+\frac{\pi}{2}\right\} .
$$

Here and henceforth we denote by $\Re(z)$ and $\Im(z)$ the real and the imaginary part of the complex number $z$, respectively. In what follows we analyze the roots of the function $f^{+}$. The roots of $f^{-}$can be treated similarly and we shall give only the final result. In order to apply Rouché's theorem, the following two lemmas give estimates for $\left|g^{+}\right|$on the contours $\Gamma_{n}^{+}(\delta)$ and for $\left|f^{+}-g^{+}\right|$in the domain $D(L)$.

Lemma 2.5. There exist $\delta_{1}>0$ and $C_{1}>0$ such that for any $\delta \in\left(0, \delta_{1}\right)$ and $N \in \mathbb{N}^{*}$ we have the following estimates

$$
\left|g^{+}(z)\right| \geq C_{1} h \delta \quad\left(z \in \Gamma_{n}^{+}(\delta), \quad 1 \leqslant n \leqslant N\right) .
$$

Proof. Firstly, we remark that the function $g^{+}$can be written in the following form

$$
g^{+}(z)=\cos (z)+\frac{1}{2}-\frac{\cos (z h)}{2} \quad(z \in \mathbb{C}) .
$$

Let $z$ be on the circle $\Gamma_{n}^{+}(\delta)$, i.e there exists $\varphi \in[0,2 \pi)$ such that $z=z_{n}^{+}+\delta e^{i \varphi}$. We replace every function of $\delta$ by its corresponding Taylor series at order $\mathrm{o}\left(\delta^{3}\right)$ and, by using several times the fact that $z_{n}^{+}$are the solutions of the equation $g^{+}(z)=0$, we deduce that

$$
\begin{aligned}
\left|g^{+}(z)\right|^{2} & =\delta^{2}\left(\sin ^{2}\left(z_{n}^{+}\right)+\frac{h^{2}}{4} \sin \left(h z_{n}^{+}\right)-h \sin \left(z_{n}^{+}\right) \sin \left(h z_{n}^{+}\right)\right)+\mathrm{o}\left(\delta^{3}\right) \\
& =\delta^{2}\left(\sin \left(z_{n}^{+}\right)-\frac{h}{2} \sin \left(h z_{n}^{+}\right)\right)^{2}+\mathrm{o}\left(\delta^{3}\right) .
\end{aligned}
$$

Finally, since $\cos \left(z_{n}^{+}\right)=-\sin ^{2}\left(\frac{h z_{n}^{+}}{2}\right)$ we have

$$
\begin{aligned}
\left|\sin \left(z_{n}^{+}\right)-\frac{h}{2} \sin \left(h z_{n}^{+}\right)\right| & \geqslant \sqrt{1-\cos ^{2}\left(z_{n}^{+}\right)}-h \sin \left(\frac{h z_{n}^{+}}{2}\right) \cos \left(\frac{h z_{n}^{+}}{2}\right) \\
& =\sqrt{\left(1-\sin ^{2}\left(\frac{h z_{n}^{+}}{2}\right)\right)\left(1+\sin ^{2}\left(\frac{h z_{n}^{+}}{2}\right)\right)}-h \sin \left(\frac{h z_{n}^{+}}{2}\right) \cos \left(\frac{h z_{n}^{+}}{2}\right) \\
& \geqslant \cos \left(\frac{h z_{n}^{+}}{2}\right)\left(1-h \sin \left(\frac{h z_{n}^{+}}{2}\right)\right) \geqslant \frac{1}{2} \cos \left(\frac{h z_{n}^{+}}{2}\right) .
\end{aligned}
$$


From (2.24), we have that

$$
\cos \left(\frac{h z_{n}^{+}}{2}\right) \geqslant \cos \left(\frac{h z_{N}^{+}}{2}\right) \geqslant \cos \left(\frac{\pi}{2}-\frac{h \pi}{4}\right) \geqslant \frac{h}{2}
$$

From the last inequality, (2.28) and (2.29) we obtain that $(2.27)$ holds on $\Gamma_{n}^{+}(\delta)$, and the proof of the lemma is complete.

Now, we pass to evaluate $\left|f^{+}(z)-g^{+}(z)\right|$ in the domain $D(L)$ defined by $(2.26)$.

Lemma 2.6. There exist $L_{0}>0, N_{1} \in \mathbb{N}^{*}$ and $C_{2}>0$ such that, for any $L>L_{0}$ and $N \geqslant N_{1}$, the following inequality holds

$$
\left|f^{+}(z)-g^{+}(z)\right| \leqslant C_{2} \exp (-L) \quad(z \in D(L)) .
$$

Proof. Firstly, from (2.23) we deduce that

$$
r(z)=\left(\sqrt{1+\sin ^{2}\left(\frac{z h}{2}\right)}+\sqrt{\sin ^{2}\left(\frac{z h}{2}\right)}\right)^{2} .
$$

Since we have that

$$
\sin \left(\frac{z h}{2}\right)=\sin \left(\frac{\Re(z) h}{2}\right) \cosh \left(\frac{\Im(z) h}{2}\right)+i \cos \left(\frac{\Re(z) h}{2}\right) \sinh \left(\frac{\Im(z) h}{2}\right),
$$

we deduce that, for any $z \in D(L)$, the following inequality holds

$$
\Re\left(\sin \left(\frac{z h}{2}\right)\right) \geqslant \sin \left(\frac{\Re(z) h}{2}\right) .
$$

Moreover, there exists $L_{0}^{\prime}>0$ and $N_{1} \in \mathbb{N}^{*}$ such that, for any $L>L_{0}^{\prime}, z \in D(L)$ and $N \geqslant N_{1}$, the following estimate is verified

$$
\Re\left(\sin ^{2}\left(\frac{z h}{2}\right)\right) \geqslant \frac{1}{2} \sin ^{2}\left(\frac{\Re(z) h}{2}\right) \geqslant 2\left|\Im\left(\sin ^{2}\left(\frac{z h}{2}\right)\right)\right| .
$$

From (2.32)-(2.33) we deduce that

$$
\Re\left(\sqrt{1+\sin ^{2}\left(\frac{z h}{2}\right)}+\sqrt{\sin ^{2}\left(\frac{z h}{2}\right)}\right) \geqslant \sqrt{1+\frac{1}{2} \sin ^{2}\left(\frac{\Re(z) h}{2}\right)}+\sin \left(\frac{\Re(z) h}{2}\right) \quad(z \in D(L)) .
$$

From (2.34) and by taking into account that

$$
\sin \left(\frac{\Re(z) h}{2}\right) \geqslant \sin \left(\frac{\pi L h}{2}\right) \geqslant L h \quad(z \in D(L)),
$$

we obtain that

$$
|r(z)| \geqslant 1+\sin \left(\frac{\Re(z) h}{2}\right) \geqslant 1+L h \quad(z \in D(L)) .
$$

Estimate (2.35) allows us to obtain (2.30). Indeed, it is easy to see that (2.35) implies that

$$
|r(z)|^{N+1} \geqslant(1+L h)^{\frac{1}{h}} \geqslant \exp (L) \quad(z \in D(L)) .
$$

Finally, from (2.22) and (2.36), we deduce that there exist $L_{0}^{\prime \prime}>0$ and $C_{2}>0$ such that the following inequalities hold for any $L>L_{0}^{\prime \prime}$

$$
\left|f^{+}(z)-g^{+}(z)\right| \leqslant \frac{C_{2}}{|r(z)|^{N+1}} \leqslant C_{2} \exp (-L) \quad(z \in D(L))
$$

and, by choosing $L_{0}=\max \left\{L_{0}^{\prime}, L_{0}^{\prime \prime}\right\}$, the proof ends. 
The following two theorems give the needed information concerning the roots of the functions $f^{ \pm}$.

Theorem 2.7. Let $\varrho>1$. There exists $\delta_{0}>0$ such that, for each $\delta \in\left(0, \delta_{0}\right)$, there exists $N_{0}(\delta) \in \mathbb{N}^{*}$ with the property that for each $N \geqslant N_{0}(\delta)$ and $n$ verifying $\varrho \ln N \leqslant n \leqslant N$, the function $f^{+}$has a unique zero $y_{n}^{+}$in $D_{n}^{+}(\delta)$.

Proof. In the following we apply Rouché's Theorem to the functions $f^{+}$and $g^{+}$given by (2.22). By using Lemma 2.5, there exist $\delta_{1}>0$ and $C_{1}>0$ such that, for each $\delta \in\left(0, \delta_{1}\right)$ and $N \in \mathbb{N}^{*}$, we have

$$
\left|g^{+}(z)\right|>C_{1} h \delta \quad\left(z \in \Gamma_{n}^{+}(\delta), 1 \leqslant n \leqslant N\right) .
$$

Moreover, from Lemma 2.6 we obtain that there exist $L_{0}>0, N_{1} \in \mathbb{N}^{*}$ and $C_{2}>0$ such that, for each $L \in\left(L_{0}, N+\frac{1}{2}\right)$ and $N \geqslant N_{1}$ we have that

$$
\left|f^{+}(z)-g^{+}(z)\right| \leqslant C_{2} \exp (-L) \quad(z \in D(L))
$$

Let $\delta_{0}=\min \left\{\delta_{1}, 1\right\}$. For any $\delta \in\left(0, \delta_{0}\right)$, there exists $N_{2}(\delta) \in \mathbb{N}$ such that, for any $N>N_{2}(\delta)$, we can find a real number $L \in\left(L_{0}, N+\frac{1}{2}\right)$ verifying

$$
L \leqslant \ln \left(\frac{2 C_{2}}{C_{1} h \delta}\right)<\varrho \ln N-1 .
$$

From (2.38) and (2.39) we deduce that

$$
\left|f^{+}(z)-g^{+}(z)\right| \leqslant \frac{C_{1} h \delta}{2} \quad(z \in D(L)) .
$$

Moreover, (2.39) implies that $D_{n}^{+}(\delta) \subset D(L)$ for any $N>N_{2}(\delta)$ and $n$ with the property that $\varrho \ln N \leqslant$ $n \leqslant N$. Consequently, from (2.37) and (2.40) we deduce that, for any $\delta \in\left(0, \delta_{0}\right)$ and $N \geqslant N_{0}:=$ $\max \left\{N_{2}(\delta), N_{1}\right\}$, the following relation holds

$$
\left|g^{+}(z)\right| \geqslant\left|f^{+}(z)-g^{+}(z)\right| \quad\left(z \in \Gamma_{n}^{+}(\delta), \varrho \ln N \leqslant n \leqslant N\right) .
$$

By applying Rouché's Theorem, we obtain that $f^{+}$vanishes only one time in the interior of $\Gamma_{n}^{+}(\delta)$, for each number $n$ which verifies $\varrho \ln N \leqslant n \leqslant N$ and the proof of the theorem is complete.

An analysis similar to the one in Theorem 2.7 shows that the following result holds.

Theorem 2.8. Let $\varrho>1$. There exists $\delta_{0}>0$ such that, for each $\delta \in\left(0, \delta_{0}\right)$, there exists $N_{0}(\delta) \in \mathbb{N}^{*}$ with the property that for each $N \geqslant N_{0}(\delta)$ and $n$ verifying $\varrho \ln N \leqslant n \leqslant N$, the function $f^{-}$has a unique zero $y_{n}^{-}$in $D_{n}^{-}(\delta)$.

The main results of this section is the following theorem which, given $\varrho>1$ and $N$ sufficiently large, localizes the eigenvalues $\left(\lambda_{n}\right)_{\varrho} \ln N \leq n \leq N$ of the matrix $A$ defined by (1.7).

Theorem 2.9. Let $\varrho>1$. There exists $\delta_{0}>0$ such that, for each $\delta \in\left(0, \delta_{0}\right)$, there exists $N_{0}(\delta) \in \mathbb{N}^{*}$ with the property that the eigenvalues $\left(\lambda_{n}\right)_{\varrho} \ln N \leqslant n \leqslant N$ of the matrix $A \in \mathcal{M}_{N}(\mathbb{R})$ with $N \geqslant N_{0}(\delta)$ are given by

$$
\lambda_{n}= \begin{cases}16 \sin ^{4}\left(\frac{y_{k}^{+} h}{2}\right) & \text { if } n=2 k+2, \\ 16 \sin ^{4}\left(\frac{y_{k}^{-} h}{2}\right) & \text { if } n=2 k+1,\end{cases}
$$

where $y_{k}^{+}$and $y_{k}^{-}$are zeros of the functions $f^{+}$and $f^{-}$respectively, localized by Theorems 2.7 and 2.8.

Proof. As mentioned at the beginning of this section, from Proposition 2.2 we deduce that the eigenvalues $\lambda$ of the matrix $A$ are of the form $16 \sin ^{4}\left(\frac{z h}{2}\right)$, where $z$ are roots of the functions $f^{+}$if $\sin (z)>0$ or $f^{-}$ if $\sin (z)<0$. Since the zeros of $f^{+}$with $\sin (z)>0$ are given by $y_{n}^{+}$with $n$ even, whereas the roots of $f^{-}$with $\sin (z)<0$ are given by $y_{n}^{-}$with $n$ odd, relations (2.42) follow immediately from Theorems 2.7 and 2.8 . 
Remark 2.10. We define for every $n \in\{1,2, \ldots, N\}$ the numbers $y_{n}$ and $z_{n}$ by

$$
y_{n}= \begin{cases}y_{n}^{+} & \text {if } n \text { is odd } \\ y_{n}^{-} & \text {if } n \text { is even }\end{cases}
$$

and

$$
z_{n}= \begin{cases}z_{n}^{+} & \text {if } n \text { is odd } \\ z_{n}^{-} & \text {if } n \text { is even }\end{cases}
$$

where we recall that $z_{n}^{ \pm}$are the roots of equations $g^{ \pm}(z)=0$ belonging to the interval $(n \pi,(n+1) \pi]$. With this notation, Theorem 2.9 says that $\lambda_{n}=16 \sin ^{4}\left(\frac{y_{n} h}{2}\right)$ and $\left|y_{n}-z_{n}\right| \leqslant \delta$ for each $\varrho \ln N \leqslant n \leqslant N$. Figure 1 depicts the graphs of the functions $\cos (z)$ and $\pm \sin ^{2}\left(\frac{z h}{2}\right)$ for $z \in[0,(N+1) \pi]$ and $N=10$. By dots we represent the roots $z_{n}$ given by (2.44).
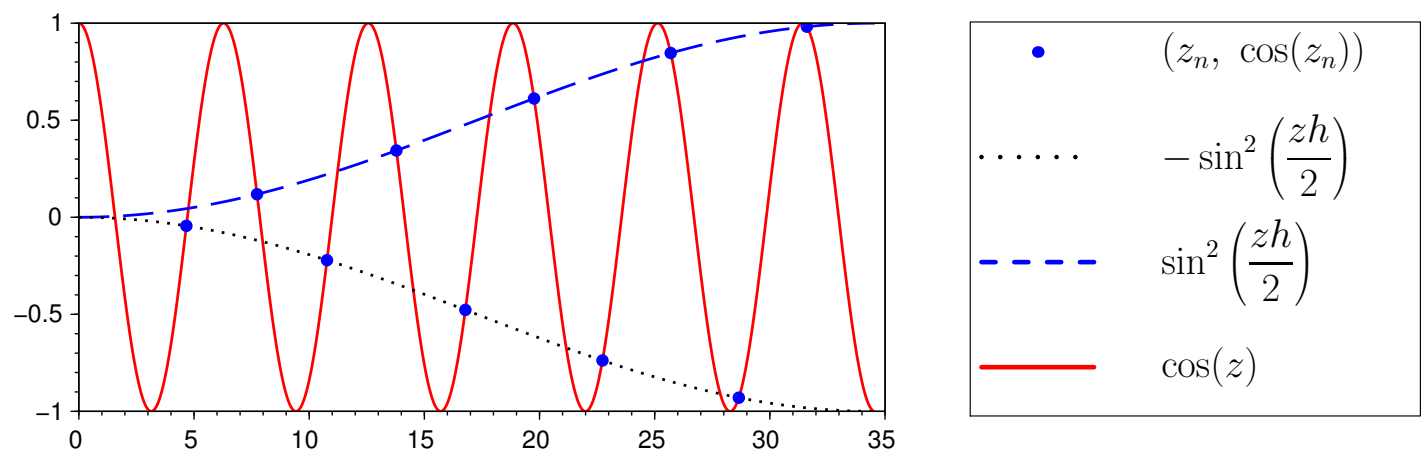

Figure 1: Solutions $z_{n}$ of equations $g^{ \pm}(z)=0$ for $N=10$.

Remark 2.11. Concerning the largest eigenvalue, $\lambda_{N}$, of the matrix $A$, we have that, for $\delta$ small enough, there exist two positive constants $\alpha_{1}$ and $\alpha_{2}$ such that the following estimate holds

$$
16\left(1-\alpha_{1} h^{2}\right)<\lambda_{N}<16\left(1-\alpha_{2} h^{2}\right) .
$$

Indeed, since $\pi N<z_{N}<\pi N+\frac{\pi}{2}$ and $\left|y_{N}-z_{N}\right| \leqslant \delta$, we obtain that, for $\delta>0$ small enough, the following estimates hold

$$
\frac{\pi}{2}-\frac{\pi\left(N-\frac{\delta}{\pi}\right)}{2(N+1)}>\frac{\pi}{2}-\frac{h y_{N}}{2}>\frac{\pi}{2}-\frac{\pi\left(N+\frac{1}{2}+\frac{\delta}{\pi}\right)}{2(N+1)},
$$

from which (2.45) follows immediately.

\subsection{Asymptotic analysis of the high eigenvectors}

Let $\lambda$ be one of the eigenvalues of the matrix $A$ given in Theorem 2.9 and let $\phi=\left(\phi_{k}\right)_{1 \leqslant k \leqslant N}$ be the normalized corresponding eigenvector. In this subsection we investigate the asymptotic behavior of $\phi_{N}$, the last component of eigenvector $\phi$. From (2.6)-(2.11) it follows that

$$
\begin{gathered}
C_{1} X_{1}^{N}=\frac{\mathfrak{C}}{X_{1} r_{N}^{1}}, \\
C_{2} X_{2}^{N}=-\frac{\mathfrak{C}}{X_{2} r_{N}^{2}}, \\
C_{3} X_{3}^{N}=\frac{\mathfrak{C}}{X_{3}}\left(-\frac{\alpha}{r_{N}^{1}}+\frac{\beta}{r_{N}^{2}}\right), \\
C_{4} X_{4}^{N}=\frac{\mathfrak{C}}{X_{4}}\left(-\frac{\beta}{r_{N}^{1}}+\frac{\alpha}{r_{N}^{2}}\right) .
\end{gathered}
$$


Hence, we have that

$$
\begin{aligned}
\phi_{N} & =C_{1} X_{1}^{N}+C_{2} X_{2}^{N}+C_{3} X_{3}^{N}+C_{4} X_{4}^{N}=\frac{\mathfrak{C}}{r_{N}^{1}}\left(\frac{1}{X_{1}}-\frac{\alpha}{X_{3}}-\frac{\beta}{X_{4}}\right)+\frac{\mathfrak{C}}{r_{N}^{2}}\left(-\frac{1}{X_{2}}+\frac{\beta}{X_{3}}+\frac{\alpha}{X_{4}}\right) \\
& =\mathfrak{C} \sqrt{\lambda}\left(\frac{1}{r_{N}^{1}}-\frac{1}{r_{N}^{2}}\right) .
\end{aligned}
$$

We recall that, the constant $\mathfrak{C}=\mathfrak{C}(\lambda)$ is chosen such that $\|\phi\|_{0}=1$. In what follows we use (2.51) to evaluate $\phi_{N}$. Useful estimates of $r_{N}^{1}$ and $r_{N}^{2}$ are given by the following lemma.

Lemma 2.12. There exists $N_{0} \in \mathbb{N}^{*}$ such that for each $N>N_{0}$ and any eigenvalue $\lambda$ of the matrix $A$ with the property that $\lambda \geqslant(3 h \ln N)^{4}$ the following estimates hold:

$$
\begin{gathered}
\frac{1}{X_{1}^{N+1}}=o(1) \sqrt{\lambda}, \\
\left|1-r_{N}^{1}\right| \leqslant\left(\frac{1}{X_{1}}\right)^{N+1}, \\
r_{N}^{2} \geqslant X_{1}^{N+1}-1 .
\end{gathered}
$$

Proof. From (2.3) it follows easily that for each $\lambda>0$ the corresponding value of $X_{1}$ satisfies

$$
X_{1}>1+\sqrt[4]{\lambda}
$$

Using the above relation, we deduce that there exists $C>0$ and $N_{0} \in \mathbb{N}$ such that

$$
X_{1}^{N+1} \geqslant e^{\frac{C \cdot \sqrt[4]{\lambda}}{h}} \quad\left(N \geqslant N_{0}\right),
$$

hence, by using that $|\lambda| \geqslant(3 h \ln N)^{4}$ we obtain that (2.52) holds. In order to deduce relations (2.53)(2.54) we remark that

$$
r_{N}^{1}=\left|\left(\frac{X_{3}}{X_{1}}\right)^{N+1}-1\right|, \quad r_{N}^{2}=\left|\left(\frac{X_{3}}{X_{2}}\right)^{N+1}-1\right|
$$

Since $\left|X_{3}\right|=\left|X_{4}\right|=1$, estimate (2.53) is justified by

$$
\left|1-r_{N}^{1}\right|=|1-|\left(\frac{X_{3}}{X_{1}}\right)^{N+1}-1|| \leqslant\left(\frac{1}{X_{1}}\right)^{N+1},
$$

whereas (2.54) is a consequence of

$$
r_{N}^{2} \geqslant||\left(\frac{X_{3}}{X_{2}}\right)^{N+1}|-1|=X_{1}^{N+1}-1 .
$$

Remark 2.13. In (2.52) and henceforth we use the classical notation o $(f(x))$ for functions of $x$ satisfying $\frac{o(f(x))}{f(x)} \rightarrow 0$ when $x$ goes to 0 . In the same spirit, the notation $O(f(x))$ stands for functions verifying $\left|\frac{O(f(x))}{f(x)}\right| \leqslant C$ when $x$ is close to 0 and for some positive constant $C$.

The following two propositions are concerned with the behavior of the constant $\mathfrak{C}$ from (2.51). Proposition 2.14 shows that, for the low eigenvalues, $\mathfrak{C}$ is bounded from below as $h$ goes to zero. Proposition 2.15 proves that, for the highest eigenvalue $\lambda_{N}$ of $A$, the corresponding constant $\mathfrak{C}$ tends to zero as $h$ goes to zero. 
Proposition 2.14. Let $\sigma \in(0,1)$. There exist $K_{0}>0$ and $N_{0} \in \mathbb{N}$ such that, for each $N \geqslant N_{0}$ and $\lambda$ an eigenvalue of the matrix $A$ with the property that $\lambda \in(\sigma, 16-\sigma)$, its associated constant $\mathfrak{C}$ verifies

$$
\mathfrak{C} \geq K_{0}
$$

Proof. From (2.12)-(2.13), we easily deduce that

$$
\Re(\alpha)=O(1), \quad \Im(\alpha)=O\left(\frac{1}{\sqrt{16-\lambda}}\right)=O(1) .
$$

Since $\lambda \in(\sigma, 16-\sigma)$, combining (2.6), (2.7) and (2.53) we obtain

$$
\begin{gathered}
C_{1} X_{1}^{k}=\frac{\mathfrak{C}}{X_{1}^{N+1-k}}+o(1) \mathfrak{C} \quad(1 \leqslant k \leqslant N), \\
C_{2} X_{2}^{k}=-\frac{\mathfrak{C}}{X_{1}^{k}}+o(1) \mathfrak{C} \quad(1 \leqslant k \leqslant N) .
\end{gathered}
$$

By using again the fact that $\lambda \in(\sigma, 16-\sigma)$, putting together (2.59) and (2.60), it follows that

$$
\left|C_{1} X_{1}^{k}+C_{2} X_{2}^{k}\right| \leqslant O(1) \mathfrak{C} \quad(1 \leqslant k \leqslant N)
$$

On the other hand, by using that $\lambda \in(\sigma, 16-\sigma),(2.47)-(2.50)$ and (2.53)-(2.54) we obtain

$$
C_{3} X_{3}^{k}+C_{4} X_{4}^{k}=-2 \Re\left(\frac{\alpha \mathfrak{C}}{X_{3}^{N+1-k}}\right)+o(1) \mathfrak{C} \quad(1 \leqslant k \leqslant N) .
$$

Hence, from (2.61)-(2.62) it follows that

$$
\left|C_{1} X_{1}^{k}+C_{2} X_{2}^{k}+C_{3} X_{3}^{k}+C_{4} X_{4}^{k}\right| \leqslant 2\left|\Re\left(\frac{\alpha \mathfrak{C}}{X_{3}^{N+1-k}}\right)\right|+O(1) \mathfrak{C} \quad(1 \leqslant k \leqslant N) .
$$

On the other hand, since $\left|\Re\left(\frac{\alpha \mathfrak{C}}{X_{3}^{N+1-k}}\right)\right| \leqslant|\alpha| \mathfrak{C}$, we obtain that there exists $C>0$ such that

$$
1=\|\phi\|_{0}^{2} \leqslant C h \sum_{k=1}^{N}\left(1+|\alpha|^{2}\right) \mathfrak{C}^{2} .
$$

Finally, since $\lambda \in(\sigma, 16-\sigma)$, from (2.58) we deduce that $|\alpha|$ is uniformly bounded in $h$. This, together with (2.64), implies that (2.57) holds.

Proposition 2.15. Within the above notation, there exist positive constants $K_{1}, K_{2}$ and $N_{0} \in \mathbb{N}^{*}$ such that for each $N \geqslant N_{0}$ the constant $\mathfrak{C}_{N}$ corresponding to $\lambda_{N}$ verifies the following estimate

$$
K_{1} h \leqslant \mathfrak{C}_{N} \leqslant K_{2} h .
$$

Proof. Let us consider $\alpha_{1}>\alpha_{2}>0$ given by (2.45). From (2.58), we easily deduce that

$$
|\alpha|=|\beta|=O\left(h^{-1}\right) .
$$

Hence, in a similar way, using (2.64) we obtain that there exists $K_{1}>0$ such that $\mathfrak{C}_{N}>K_{1} h$.

For every $h>0$, using (2.45), there exists $K \in\left(\alpha_{2}, \alpha_{1}\right)$ such that $\lambda_{N}=16-K h^{2}$. Then, from (2.4) we obtain the following asymptotic expansion $X_{3}=-1+i \sqrt{\frac{K}{8}} h+o\left(h^{2}\right)$, thus for small enough $h$ and for $1 \leqslant k \leqslant \delta N$, with $\delta \in(0,1)$, we have

$$
X_{3}^{N+1-k}=\left(-1+i \sqrt{\frac{K}{8}} h+o\left(h^{2}\right)\right)^{N+1-k}=(-1)^{N+1-k} e^{-i \sqrt{\frac{K}{8}}(N+1-k) h+o(h)} .
$$


Evaluating the term in the right-hand side of (2.63), we obtain

$$
\begin{aligned}
\left|\Re\left(\frac{\alpha}{X_{3}^{N+1-k}}\right)\right| & =\left|\Re\left(\frac{\frac{1}{2}\left(1-i \frac{R_{+}}{R_{-}}\right)}{e^{-i \sqrt{\frac{K}{8}}(N+1-k) h+o(h)}}\right)\right| \\
& =\frac{1}{2} \cos \left(\sqrt{\frac{K}{8}(N+1-k) h}\right)+\frac{1}{2} \sin \left(\sqrt{\frac{K}{8}(N+1-k) h}\right) \sqrt{\frac{4+\sqrt{\lambda_{N}}}{4-\sqrt{\lambda_{N}}}+o(h) .}
\end{aligned}
$$

By choosing $0<\delta_{1}<\delta_{2}<1$ such that there exist $C_{1}>0$ which verifies $\sin \left(\sqrt{\frac{K}{8}(N+1-k) h}\right)>C_{1}$, for each $n \in\left(\delta_{1} N, \delta_{2} N\right)$, from the above relation combined to (2.45) it follows that there exists $C_{2}>0$ such that

$$
\left|\Re\left(\frac{\alpha}{X_{3}^{N+1-k}}\right)\right|=\frac{2}{C_{2} h}+O(1) \quad\left(\delta_{1} N \leq k \leq \delta_{2} N\right) .
$$

From (2.63)-(2.67) we have that

$$
\begin{aligned}
1=\|\phi\|_{0}^{2}=h \sum_{k=1}^{N}\left|C_{1} X_{1}^{k}+C_{2} X_{2}^{k}+C_{3} X_{3}^{k}+C_{4} X_{4}^{k}\right|^{2} \\
\qquad h \sum_{k=\delta_{1} N}^{\delta_{2} N}\left|C_{1} X_{1}^{k}+C_{2} X_{2}^{k}+C_{3} X_{3}^{k}+C_{4} X_{4}^{k}\right|^{2} \geqslant h \sum_{k=\delta_{1} N}^{\delta_{2} N} \frac{2 \mathfrak{C}^{2}}{C_{2}^{2} h^{2}}=\frac{\left(\delta_{2}-\delta_{1}\right) \mathfrak{C}^{2}}{C_{2}^{2} h^{2}},
\end{aligned}
$$

and finally $\mathfrak{C} \leq K_{2} h$, where $K_{2}=\frac{C_{2}}{\sqrt{\delta_{2}-\delta_{1}}}$.

Now we have all the ingredients needed to evaluate $\phi_{N}$. The following result holds true.

Theorem 2.16. Let $\sigma \in(0,1)$. There exist $K>0$ and $N_{0} \in \mathbb{N}^{*}$ such that, for each $N \geqslant N_{0}$ and each $\lambda$ eigenvalue of the matrix $A$ with the property that $\lambda \in(\sigma, 16-\sigma)$, the corresponding normalized eigenvector $\phi=\left(\phi_{k}\right)_{1 \leqslant k \leqslant N} \in \mathbb{R}^{N}$ has the following property

$$
\left|\phi_{N}\right|>K \sqrt{\lambda}
$$

Moreover, if $\phi^{N} \in \mathbb{R}^{N}$ is the eigenvector corresponding to the last eigenvalue $\lambda_{N}$, we have that

$$
\frac{\left|\phi_{N}^{N}\right|}{\sqrt{\lambda_{N}}}=O(h)
$$

Proof. Inequality (2.68) is a consequence of $(2.51),(2.53),(2.54)$ and (2.57). On the other hand, taking into account (2.65), we deduce that (2.69) holds for the normalized eigenvector corresponding to the last eigenvalue $\lambda_{N}$.

Remark 2.17. Inequality (2.68) shows that a uniform observability inequality holds for the eigenvectors corresponding to eigenvalues $\lambda \in(\sigma, 16-\sigma)$. Moreover, according to (2.69), the observability inequality for the highest eigenmodes degenerates. On the other hand, for the low frequencies $\lambda \leqslant \sigma$, our asymptotic estimates does not allow to obtain useful estimates for $\phi_{N}$. This case will be treated in a following section. In Figure 2 is displayed the evolution of the quantity $\frac{\left(\phi_{N}^{N}\right)^{2}}{\lambda_{N}}$ as a function of $N$ (and respectively $h$ ). We remark that $\frac{\left(\phi_{N}^{N}\right)^{2}}{\lambda_{N}} \approx C_{0} h^{2}$ with $C_{0}=2.2$ We mention that the eigenvalues and eigenvectors of the matrix A were numerically approximated.

\subsection{Numerical estimates of the low frequencies}

This section is devoted to analyze the behavior of the low eigenvalues of the matrix $A$ for which Theorem 2.9 does not offer any information. This will be done by using the fact that the low eigenvalues of the operator $A_{h}=\frac{1}{h^{4}} A$ approximate the corresponding low eigenvalues of the differential operator of the 


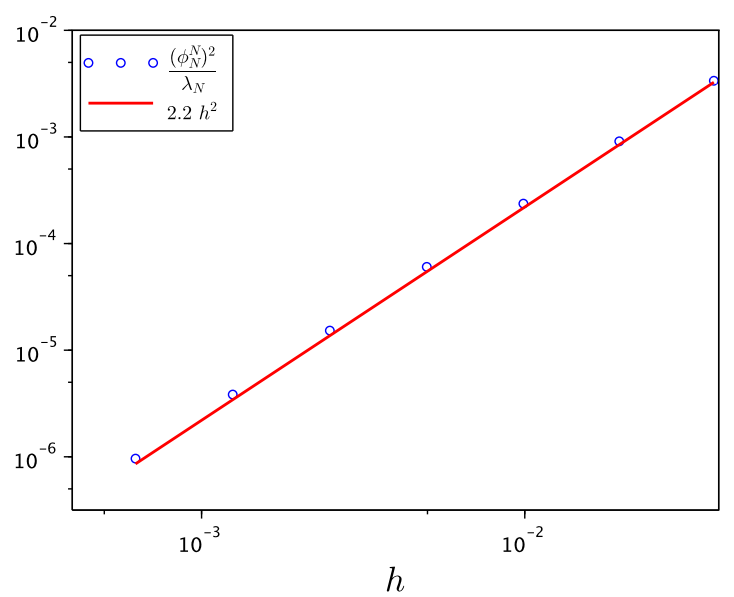

Figure 2: Evolution of the quantity $\frac{\left(\phi_{N}^{N}\right)^{2}}{\lambda_{N}}$ as a function of $h$.

beam equation (1.4). Let $(\widetilde{A}, D(\widetilde{A}))$ be the unbounded elliptic operator in $L^{2}(0,1)$ associated to the clamped beam equation and defined by

$$
\left\{\begin{array}{l}
\widetilde{A} u=\partial_{x}^{4} u \quad(u \in D(\widetilde{A})), \\
D(\widetilde{A})=H^{4}(0,1) \cap H_{0}^{2}(0,1) .
\end{array}\right.
$$

The operator $(\widetilde{A}, D(\widetilde{A}))$ has a sequence of simple positive eigenvalues $\left(\widetilde{\lambda}_{n}\right)_{n \geqslant 1}$ of the form

$$
\tilde{\lambda}_{n}=\left(n+\frac{1}{2}\right)^{4} \pi^{4}+v_{n} \quad(n \geqslant 1),
$$

where $\left(v_{n}\right)_{n \geqslant 1}$ is a sequence converging exponentially to zero (see [11, Lemma 6.10.2]). Any eigenfunction $\widetilde{\varphi}_{n}$ corresponding to $\widetilde{\lambda}_{n}$ verifies $\widetilde{\varphi}_{n} \in \mathcal{C}^{\infty}(0,1) \cap D(\widetilde{A})$.

Let $N \in \mathbb{N}^{*}, h=\frac{1}{N+1}$ and $x_{j}=j h, 0 \leqslant j \leqslant N$, be an equidistant division of the interval $[0,1]$. The following lemma is a classical result concerning the error in the finite-differences approximation of the operator $(\widetilde{A}, D(\widetilde{A}))$.

Lemma 2.18. There exists an absolute constant $C>0$ such that, for any $u \in \mathcal{C}^{6}[0,1] \cap D(\widetilde{A})$, we have that

$$
\left|(\widetilde{A} u)\left(x_{j}\right)-\left(A_{h} U\right)_{j}\right| \leqslant C h^{2} \sup _{x \in[0,1]}\left|u^{(6)}(x)\right| \quad(1 \leqslant j \leqslant N),
$$

where $U=\left(\begin{array}{c}u\left(x_{1}\right) \\ \cdot \\ \cdot \\ \cdot \\ u\left(x_{N}\right)\end{array}\right)$.

We recall that the matrix $A_{h}$ has a family of eigenvalues $\left(\frac{\lambda_{n}}{h^{4}}\right)_{1 \leqslant n \leqslant N}$. The following theorem is a consequence of a well known result concerning the approximation of the eigenvalues of an elliptic operator (see [8, Theorem 3.1]).

Theorem 2.19. Let $\varepsilon \in(0,2)$. There exist $N_{0}>0$ and $C>0$ such that, for each $N \geq N_{0}$, the following estimate holds:

$$
\left|\widetilde{\lambda}_{n}-\frac{\lambda_{n}}{h^{4}}\right| \leq C h^{\varepsilon} \quad\left(1 \leq n \leq N^{\frac{1}{6}(2-\varepsilon)}\right) .
$$


Proof. Let $\widetilde{\lambda}_{n}$ be an eigenvalue of the continuous operator $(\widetilde{A}, D(\widetilde{A}))$ and let $\widetilde{\varphi}_{n}$ be a corresponding eigenfunction. From (2.72), we deduce that

$$
A_{h} \widetilde{\Phi}_{n}-\widetilde{\lambda}_{n} \widetilde{\Phi}_{n}=\widetilde{\Psi}_{n} \in \mathbb{R}^{N} \text { with }\left\|\widetilde{\Psi}_{n}\right\|_{\infty} \leqslant C h^{2} \sup _{x \in[0,1]}\left|\widetilde{\varphi}_{n}^{(6)}(x)\right|,
$$

where $\widetilde{\Phi}_{n}=\left(\begin{array}{c}\widetilde{\varphi}_{n}\left(x_{1}\right) \\ \cdot \\ \cdot \\ \cdot \\ \widetilde{\varphi}_{n}\left(x_{N}\right)\end{array}\right)$ will be normalized such that

$$
\left\|\widetilde{\Phi}_{n}\right\|_{0}:=\sqrt{h \sum_{j=1}^{N}\left|\widetilde{\varphi}_{n}\left(x_{j}\right)\right|^{2}}=1 .
$$

Since $A_{h}$ is a symmetric matrix, it follows that it is diagonalisable and there exist a unitary matrix $P \in \mathcal{M}_{N}(\mathbb{R})$ and a diagonal matrix $D_{h} \in \mathcal{M}_{N}(\mathbb{R})$ such that

$$
P^{-1} A_{h} P=D_{h} .
$$

Of course, the elements on the diagonal of $D_{h}$ are the eigenvalues of the matrix $A_{h}$ which can be supposed ordered in a strictly increasing sequence $\left(\frac{\lambda_{j}}{h^{4}}\right)_{1 \leq j \leq N}$. From(2.74) and (2.76) we deduce that

$$
\widetilde{\Phi}_{n}=\left(A_{h}-\widetilde{\lambda}_{n} \mathcal{I}_{h}\right)^{-1} \widetilde{\Psi}_{n}=P\left(D_{h}-\widetilde{\lambda}_{n} \mathcal{I}_{h}\right)^{-1} P^{-1} \widetilde{\Psi}_{n} .
$$

Since $P$ is unitary (and thus $\|P\|_{0}=\left\|P^{-1}\right\|_{0}=1$ ), it follows from (2.77) and (2.75) that

$$
\min _{1 \leqslant j \leqslant N}\left|\widetilde{\lambda}_{n}-\frac{\lambda_{j}}{h^{4}}\right| \leqslant\left\|\widetilde{\Psi}_{n}\right\|_{0}
$$

By taking into account that the sequence $\left(\frac{\lambda_{j}}{h^{4}}\right)_{1 \leq j \leq N}$ is strictly increasing, from (2.74) and the last relation, we deduce that

$$
\left|\widetilde{\lambda}_{n}-\frac{\lambda_{n}}{h^{4}}\right| \leqslant C h^{2} \sup _{x \in[0,1]}\left|\widetilde{\varphi}_{n}^{(6)}(x)\right| .
$$

From the known form of the eigenfunction $\widetilde{\varphi}_{n}$ (see (6.10.13) in [11, Lemma 6.10.2]) a straightforward computation allows us to show that there exists a positive constant $C$ such that

$$
\sup _{x \in[0,1]}\left|\widetilde{\varphi}_{n}^{(6)}(x)\right| \leqslant C n^{6} \quad(1 \leqslant n \leqslant N) .
$$

By combining (2.78) and (2.79), (2.73) follows immediately.

A direct consequence of Theorem 2.19 is the following result which ensures the existence of a positive gap between two consecutive discrete low eigenvalues $\lambda_{n}$.

Corollary 2.20. Let $\varepsilon \in(0,2)$. There exist $N_{0}>0$ and $d>0$ such that, for each $N \geq N_{0}$, the following estimate holds:

$$
\frac{1}{h^{2}}\left|\sqrt{\lambda_{n+1}}-\sqrt{\lambda_{n}}\right| \geqslant d n \quad\left(1 \leq n \leq N^{\frac{1}{6}(2-\varepsilon)}\right) .
$$

Proof. Since the eigenvalues $\left(\widetilde{\lambda}_{n}\right)_{n \geqslant 1}$ of the continuous operator verify (2.71), there exists a constant $d>0$ such that

$$
\left|\sqrt{\widetilde{\lambda}_{n+1}}-\sqrt{\tilde{\lambda}_{n}}\right| \geq 2 d n \quad(n \geq 1) .
$$

The desired result (2.80) follows easily by taking into account that

$$
\frac{1}{h^{2}}\left|\sqrt{\lambda_{n+1}}-\sqrt{\lambda_{n}}\right| \geqslant\left|\sqrt{\widetilde{\lambda}_{n+1}}-\sqrt{\widetilde{\lambda}_{n}}\right|-\left|\frac{\sqrt{\lambda_{n+1}}}{h^{2}}-\sqrt{\widetilde{\lambda}_{n+1}}\right|-\left|\frac{\sqrt{\lambda_{n}}}{h^{2}}-\sqrt{\tilde{\lambda}_{n}}\right|,
$$

and by using estimate (2.73) in order to bound by $d$ the sum of the last two terms. 


\subsection{An observability inequality for the low eigenvectors}

The aim of this section is to prove an observability inequality for the low eigenvectors of the matrix $A_{h}$ similar to (2.68). We use a discrete multiplier method already presented in [2]. This method allows us to show that, for every $0<\sigma<16$, given an eigenvalue $\lambda$ of the matrix $A$ with the property $\lambda \leqslant \sigma$ and given a normalized eigenvector $\phi=\left(\phi_{k}\right)_{1 \leqslant k \leqslant N}$ corresponding to $\lambda$, the following observability inequality for eigenvectors holds

$$
\left|\phi_{N}\right| \geqslant K \sqrt{\lambda}
$$

where $K$ is a positive constant independent of $h$ or $N$. We recall that, inequality (2.81) has been obtained for the eigenvectors corresponding to eigenvalues $\lambda \in(\sigma, 16-\sigma)$. This section shows that the same estimate holds for the low eigenvalues $\lambda \leqslant \sigma$.

Since we use the discrete multiplier method, let us define in $\mathcal{M}_{N}(\mathbb{R})$ the following matrices discretizing the third $\left(D_{3}\right)$ and the first $\left(D_{1 c}, D_{1 b}\right)$ derivatives, respectively

$$
D_{3}=\left(\begin{array}{rrrrrrrrr}
3 & -3 & 1 & 0 & \ldots & \ldots & \ldots & \ldots & 0 \\
-1 & 3 & -3 & 1 & 0 & \ldots & \ldots & \ldots & 0 \\
0 & -1 & 3 & -3 & 1 & 0 & \ldots & \ldots & 0 \\
0 & 0 & -1 & 3 & -3 & 1 & 0 & \ldots & 0 \\
\vdots & \ddots & \ddots & \ddots & \ddots & \ddots & \ddots & \ddots & \vdots \\
0 & \ldots & 0 & 0 & -1 & 3 & -3 & 1 & 0 \\
0 & \ldots & \ldots & 0 & 0 & -1 & 3 & -3 & 1 \\
0 & \ldots & \ldots & \ldots & 0 & 0 & -1 & 3 & -3 \\
0 & \ldots & \ldots & \ldots & \ldots & 0 & 0 & -1 & 4
\end{array}\right)
$$

and

$$
D_{1 c}=\left(\begin{array}{rrrrrrr}
0 & 1 & 0 & \ldots & \ldots & \ldots & 0 \\
-1 & 0 & 1 & 0 & \ldots & \ldots & 0 \\
0 & -1 & 0 & 1 & 0 & \ldots & 0 \\
\vdots & \ddots & \ddots & \ddots & \ddots & \ddots & \vdots \\
0 & \ldots & 0 & -1 & 0 & 1 & 0 \\
0 & \ldots & \ldots & 0 & -1 & 0 & 1 \\
0 & \ldots & \ldots & \ldots & 0 & -1 & 0
\end{array}\right), \quad D_{1 b}=\left(\begin{array}{rrrrrrr}
1 & 0 & 0 & \ldots & \ldots & \ldots & 0 \\
-1 & 1 & 0 & 0 & \ldots & \ldots & 0 \\
0 & -1 & 1 & 0 & 0 & \ldots & 0 \\
\vdots & \ddots & \ddots & \ddots & \ddots & \ddots & \vdots \\
0 & \ldots & 0 & -1 & 1 & 0 & 0 \\
0 & \ldots & \ldots & 0 & -1 & 1 & 0 \\
0 & \ldots & \ldots & \ldots & 0 & -1 & 1
\end{array}\right) .
$$

A straightforward computation gives the following lemma.

Lemma 2.21. With the above notation we have that

1. $A=D_{1 b} D_{3}+\left(\begin{array}{rrrrr}4 & -1 & 0 & \ldots & 0 \\ 0 & 0 & 0 & \ldots & 0 \\ \vdots & \ddots & \ddots & \ddots & \vdots \\ 0 & \ldots & 0 & 0 & 0\end{array}\right)$,
2. $D_{3}=D_{1 b}^{\prime} B+\left(\begin{array}{rrrrr}0 & 0 & 0 & \ldots & 0 \\ 0 & 0 & 0 & \ldots & 0 \\ \vdots & \ddots & \ddots & \ddots & \vdots \\ 0 & \ldots & 0 & 0 & 2\end{array}\right)$,
3. $B=D_{1 b} D_{1 b}^{\prime}+\left(\begin{array}{ccccc}1 & 0 & 0 & \ldots & 0 \\ 0 & 0 & 0 & \ldots & 0 \\ \vdots & \ddots & \ddots & \ddots & \vdots \\ 0 & \ldots & 0 & 0 & 0\end{array}\right)$,

4. $D_{1 b}^{\prime}(v \cdot w)=D_{1 b}^{\prime} v \cdot w+S_{0}^{\prime} v \cdot D_{1 b}^{\prime} w$, for every vectors $v, w \in \mathbb{R}^{N}$, where

$$
S_{0}=I-D_{1 b}
$$

where $I$ denotes the identity matrix in $\mathcal{M}_{N}(\mathbb{R})$. 
In the remaining part of this section we denote by "." the product component by component of two vectors in $\mathbb{R}^{N}$, i.e., for any two vectors $U, V \in \mathbb{R}^{N}$ we have that $U . V$ is the vector in $\mathbb{R}^{N}$ whose $j$-th component is given by $(U . V)_{j}=u_{j} v_{j}$.

Remark 2.22. Note that, we obtain an observability estimate for the eigenvectors of the matrix $\bar{A}=B^{2}$. This would give the observability inequality for the semi-discrete problem studied in [10], when we consider an eigenfunction of the system as initial data. Let $\bar{\phi} \in \mathbb{R}^{N}$ be a normalized eigenvector corresponding to the eigenvalue $\bar{\lambda}$ of the matrix $\bar{A}$. Firstly, we remark that $\bar{\phi}$ is also an eigenvector of the matrix $B$, corresponding to the eigenvalue $\bar{\lambda}^{\frac{1}{2}}$. Therefore, we can use the identity proved in [7, Lemma 1.1] to show that following relation holds

$$
\bar{\phi}_{N}^{2}=\frac{1}{2}\left(4-\bar{\lambda}^{\frac{1}{2}}\right)\langle B \bar{\phi}, \bar{\phi}\rangle
$$

Hence, (2.83) implies that $\bar{\phi}_{N}^{2}$ is of order of $\langle B \bar{\phi}, \bar{\phi}\rangle$ (or, equivalently, of order of $\bar{\lambda}^{\frac{1}{2}}$ ), as long as $\bar{\lambda}$ is not approaching 16. In order to ensure that, one has to filter the high frequencies. This remark is the main ingredient for proving the nonuniform observability result [10, Theorem 2.1].

The main difficulty comes from the fact that, when we are dealing with the matrix $A$, instead of $\bar{A}$, we cannot reduce the problem to a similar one for the matrix $B$ as in the previous remark. The following result gives an identity for the eigenvectors of the matrix $A$ we are concerned with. We are inspired on the multiplier method used to prove the observability inequality (1.3) for the continuous equation (1.4).

Lemma 2.23. Let $N \in \mathbb{N}^{*}$ and let $\phi=\left(\phi_{k}\right)_{1 \leqslant k \leqslant N}$ be the normalized eigenvector of $A$ corresponding to the eigenvalue $\lambda$. Then the following identity holds

$$
\phi_{N}^{2}=\langle A \phi, \phi\rangle-\frac{\lambda}{4}\langle B \phi, \phi\rangle-\frac{h}{4}\left(4 \phi_{1}^{2}+4 \phi_{N}^{2}-\phi_{1} \phi_{2}-\phi_{N-1} \phi_{N}\right) .
$$

Proof. Let $J=(1,2, \ldots, N)^{\prime}$. Relation (2.84) can be deduced multiplying the relation $A \phi=\lambda \phi$ by $J . D_{1 c} \phi$. We evaluate first the product $\left\langle\phi, J . D_{1 c} \phi\right\rangle$ and we obtain that

$$
\left\langle\phi, J . D_{1 c} \phi\right\rangle=\left\langle D_{1 c} \phi . \phi, J\right\rangle=\left\langle\left(-S_{0}+S_{0}^{\prime}\right) \phi . \phi, J\right\rangle .
$$

Since

$$
D_{1 b}^{\prime}\left(S_{0} \phi . \phi\right)=\left(I-S_{0}^{\prime}\right)\left(S_{0} \phi . \phi\right)=S_{0} \phi . \phi-S_{0}^{\prime}\left(S_{0} \phi . \phi\right)=S_{0} \phi . \phi-S_{0}^{\prime} \phi . \phi,
$$

the above relation becomes

$$
\begin{aligned}
\left\langle\phi, J . D_{1 c} \phi\right\rangle & =-\left\langle D_{1 b}^{\prime}\left(S_{0} \phi . \phi\right), J\right\rangle=-\left\langle S_{0} \phi . \phi, D_{1 b} J\right\rangle \\
& =-\left\langle S_{0} \phi, \phi\right\rangle=-\frac{1}{2}\left(\left\langle S_{0} \phi, \phi\right\rangle+\left\langle S_{0}^{\prime} \phi, \phi\right\rangle\right) \\
& =\left\langle-\frac{1}{2}\left(S_{0}+S_{0}^{\prime}\right) \phi, \phi\right\rangle=\left\langle\left(\frac{1}{2} B-I\right) \phi, \phi\right\rangle .
\end{aligned}
$$

Hence, it follows that

$$
\left\langle\phi, J . D_{1 c} \phi\right\rangle=\frac{1}{2}\langle B \phi, \phi\rangle-\langle\phi, \phi\rangle .
$$

We evaluate the product $\left\langle A \phi, J . D_{1 c} \phi\right\rangle$ by using the first assertion of Lemma 2.21 and it follows that

$$
\left\langle A \phi, J . D_{1 c} \phi\right\rangle=\left\langle D_{1 b} D_{3} \phi, J . D_{1 c} \phi\right\rangle+h\left(4 \phi_{1} \phi_{2}-\phi_{2}^{2}\right) .
$$

By using the point (4) of Lemma 2.21, we have that

$$
\begin{aligned}
\left\langle D_{1 b} D_{3} \phi, J . D_{1 c} \phi\right\rangle & =\left\langle D_{3} \phi, D_{1 b}^{\prime}\left(J . D_{1 c}\right) \phi\right\rangle \\
& =\left\langle D_{3} \phi, D_{1 b}^{\prime} J . D_{1 c} \phi+S_{0}^{\prime} J . D_{1 b}^{\prime}\left(D_{1 c} \phi\right)\right\rangle \\
& =\left\langle D_{3} \phi,-D_{1 c} \phi+S_{0}^{\prime} J . D_{1 b}^{\prime}\left(D_{1 c} \phi\right)\right\rangle-(N+1) \phi_{N-1}\left(4 \phi_{N}-\phi_{N-1}\right) .
\end{aligned}
$$


Moreover, we deduce that

$$
\left\langle D_{3} \phi, D_{1 c} \phi\right\rangle=-\langle A \phi, \phi\rangle+\left\langle B \phi, D_{1 b}^{2} \phi\right\rangle+\left(4 \phi_{1}-\phi_{2}\right) \phi_{1}+2 \phi_{N}\left(\phi_{N}-\phi_{N-1}\right)
$$

and

$$
\left\langle D_{3} \phi, S_{0}^{\prime} J . D_{1 b}^{\prime}\left(D_{1 c} \phi\right)\right\rangle=\langle A \phi, \phi\rangle-2 \phi_{1}^{2}-2 \phi_{N}^{2}+\left(2 v_{1}-v_{2}\right)^{2}-(N+1)\left(-v_{N-1}+2 v_{N}\right)^{2} .
$$

The above relations combined to the third relation in Lemma 2.21 allow us to deduce the following expression for the product $\left\langle A \phi, J . D_{1 c} \phi\right\rangle$ :

$$
\left\langle A \phi, J . D_{1 c} \phi\right\rangle=2\langle A \phi, \phi\rangle+\left\langle B \phi, S_{0} B \phi\right\rangle-4 \phi_{N}^{2}-h\left(4 \phi_{N}^{2}+4 \phi_{1}^{2}-2 \phi_{1} \phi_{2}-2 \phi_{N} \phi_{N-1}\right) .
$$

Finally, since $\phi$ is the eigenvector of $A$ corresponding to the eigenvalue $\lambda$, we obtain that

$$
\left\langle B \phi, S_{0} B \phi\right\rangle=\langle A \phi, \phi\rangle-\frac{\lambda}{2}\langle B \phi, \phi\rangle-h\left(\phi_{1} \phi_{2}+\phi_{N} \phi_{N-1}\right)
$$

and it follows that

$$
\left\langle A \phi, J . D_{1 c} \phi\right\rangle=3\langle A \phi, \phi\rangle-\frac{\lambda}{2}\langle B \phi, \phi\rangle-4 \phi_{N}^{2}-h\left(4 \phi_{1}^{2}+4 \phi_{N}^{2}-\phi_{1} \phi_{2}-\phi_{N-1} \phi_{N}\right) .
$$

Relation (2.84) can be easily deduced from (2.85)-(2.86) and the proof of the lemma is complete.

By comparing (2.84) to (2.83), it is not so easy to see that $\phi_{N}^{2}$ is bounded from below by its corresponding eigenvalue $\lambda$. However, we are able to show that this is indeed the case, at least for the low frequencies.

Theorem 2.24. Let $N \in \mathbb{N}^{*}, \sigma \in(0,1)$ and $\phi=\left(\phi_{k}\right)_{1 \leqslant k \leqslant N}$ be the normalized eigenvector of $A$ corresponding to the eigenvalue $\lambda \in(0,16-\sigma)$. Then there exists a constant $K>0$, independent of $N$ and $\lambda$, such that the following estimate holds

$$
\left|\phi_{N}\right| \geqslant K \sqrt{\lambda}
$$

Proof. Let us remark that

$$
\begin{aligned}
\|B \phi\|_{0}^{2} & =\langle B \phi, B \phi\rangle=\left\langle B^{2} \phi, \phi\right\rangle=\langle A \phi, \phi\rangle-2 h\left(\phi_{1}^{2}+\phi_{N}^{2}\right) \\
& =\lambda\langle\phi, \phi\rangle-2 h\left(\phi_{1}^{2}+\phi_{N}^{2}\right) .
\end{aligned}
$$

Hence, the following inequalities hold

$$
\|B \phi\|_{0} \leqslant \sqrt{\lambda}
$$

and

$$
h\left(2 \phi_{1}-\phi_{2}\right)^{2}+h\left(2 \phi_{N}-\phi_{N-1}\right)^{2} \leqslant \lambda .
$$

Estimate (2.84) can be written as

$$
\phi_{N}^{2}=\frac{\lambda}{4}(4\langle\phi, \phi\rangle-\langle B \phi, \phi\rangle)-\frac{h}{2}\left(\phi_{1}^{2}+\phi_{N}^{2}\right)-\frac{h}{4}\left(\left(2 \phi_{1}-\phi_{2}\right) \phi_{1}+\left(2 \phi_{N}-\phi_{N-1}\right) \phi_{N}\right) .
$$

Combining the above relation with the inequalities

$$
\begin{aligned}
2\left(2 \phi_{1}-\phi_{2}\right) & \leq \varsigma\left(2 \phi_{1}-\phi_{2}\right)^{2}+\frac{1}{\varsigma} \phi_{1}^{2} \\
2\left(2 \phi_{N}-\phi_{N-1}\right) & \leq \varsigma\left(2 \phi_{N}-\phi_{N-1}\right)^{2}+\frac{1}{\varsigma} \phi_{N}^{2},
\end{aligned}
$$

for any $\varsigma>0$, gives

$$
\phi_{N}^{2} \geqslant \frac{\lambda}{4}\left(4\|\phi\|_{0}^{2}-\|B \phi\|_{0}\|\phi\|_{0}\right)-\frac{h}{2}\left(1+\frac{1}{4 \varsigma}\right)\left(\phi_{1}^{2}+\phi_{N}^{2}\right)-\frac{\varsigma h}{8}\left(\left(2 \phi_{1}-\phi_{2}\right)^{2}+\left(2 \phi_{N}-\phi_{N-1}\right)^{2}\right) .
$$


By using (2.88) and (2.89) and the fact that $\|\phi\|_{0}=1$, we deduce from the last inequality that, for any $\varsigma>0$, we have

$$
\frac{h}{2}\left(1+\frac{1}{4 \varsigma}\right)\left(\phi_{1}^{2}+\phi_{N}^{2}\right)+\phi_{N}^{2} \geqslant \frac{\lambda}{4}(4-\sqrt{\lambda})-\frac{\varsigma \lambda}{8} .
$$

Since $\lambda \in(0,16-\sigma)$, by considering $\varsigma=4-\sqrt{16-\sigma}>0$ in estimate $(2.90)$, we deduce that

$$
\frac{h}{2}\left(1+\frac{1}{4 \varsigma}\right)\left(\phi_{1}^{2}+\phi_{N}^{2}\right)+\phi_{N}^{2} \geqslant \frac{\lambda}{8}(4-\sqrt{16-\sigma}) .
$$

Finally, by using that $\phi_{1}^{2}=\phi_{N}^{2}$ (from the symmetry), we obtain from (2.91) that there exists a constant $K>0$ given by $K=\frac{\sqrt{4-\sqrt{16-\sigma}}}{4}$, such that (2.87) holds and the proof of the proposition is complete.

Remark 2.25. In addition to Theorem 2.16 and as in the case treated in [10], we obtain the desired observability inequality for eigenvectors holds if the highest frequencies are filtered and $\lambda \in(0,16-\sigma)$ with $\sigma \in(0,1)$.

\section{Observability results}

This section gives the main observability result concerning the solutions of (1.12). In order to give the Fourier expansion of the solutions $\left(\begin{array}{c}Y_{h} \\ \dot{Y}_{h}\end{array}\right)$ of $(1.12)$ we remark that the set $\left(\Phi^{n}\right)_{1 \leqslant|n| \leqslant N}$ defined by

$$
\Phi^{n}=\left(\begin{array}{c}
\frac{h^{2}}{\sqrt{\lambda_{|n|}}} \\
-\operatorname{sgn}(n) i
\end{array}\right) \phi^{|n|} \quad(1 \leqslant|n| \leqslant N),
$$

forms an orthonormal basis in $\mathbb{C}^{2 N}$ with respect to the inner product (1.10). We recall that, according to Proposition 2.1, the set $\left(\phi^{n}\right)_{1 \leqslant n \leqslant N}$ is an orthonormal basis for $\mathbb{C}^{N}$. Let $\gamma \in(0,1)$. We define the following space of filtered data

$$
\mathcal{C}_{h}(\gamma)=\left\{\left(\begin{array}{c}
Y_{h}^{0} \\
Y_{h}^{1}
\end{array}\right)=\sum_{1 \leqslant|n| \leqslant \gamma N} a_{n} \Phi^{n}, \quad\left(a_{n}\right)_{1 \leqslant|n| \leqslant \gamma N} \subset \mathbb{C}\right\} .
$$

We remark that the condition $|n| \leqslant \gamma N$ in the definition of the space $\mathcal{C}_{h}(\gamma)$ is equivalent to the fact that the eigenfrequencies $\left|\lambda_{n}\right| \geqslant C / h^{4}$, for some $C=C(\gamma)>0$, are filtered out. The following result, concerning the separation of the eigenvalues of our matrix $A$, will allow us to prove the desired observability inequality.

Proposition 3.1. Let $T>0$. There exist $N_{0}, n_{T} \in \mathbb{N}^{*}$ such that, for any $N \geqslant N_{0}$, the eigenvalues $\lambda_{n}$ of the matrix A verify

$$
\sqrt{\lambda_{n+1}}-\sqrt{\lambda_{n}} \geqslant \frac{2 \pi}{T} h^{2} \quad\left(n_{T} \leqslant n \leqslant N-n_{T}\right)
$$

Proof. Let $\left(y_{n}\right)_{n}$ be the family given by (2.43). Let $\varrho>1$ and let $\delta_{0}$ be given by Theorem 2.9. For any $\delta \in\left(0, \delta_{0}\right)$ there exists $N_{0}(\delta)$ such that $\left|y_{n}-z_{n}\right| \leqslant \delta$ for $\varrho \ln N \leqslant n \leqslant N$ and $N \geqslant N_{0}(\delta)$. Hence,

$$
\left|y_{n+1}-y_{n}\right| \geqslant\left|z_{n+1}-z_{n}\right|-2 \delta \quad(\varrho \ln N \leqslant n \leqslant N-1) .
$$

According to Lemma 2.3 and relations (2.24), it follows that

$$
\left|z_{n+1}-z_{n}\right| \geqslant \frac{\pi}{2}
$$

By choosing $\delta<\min \left\{\delta_{0}, \frac{\pi}{8}\right\}$, from (3.3) and (3.4), we deduce that, for each $N \geqslant N_{0}(\delta)$,

$$
\left|y_{n+1}-y_{n}\right| \geqslant \frac{\pi}{4} \quad(\varrho \ln N \leqslant n \leqslant N-1) .
$$


Now, for each $\varrho N \leqslant n \leqslant N-1$, there exists $\xi_{n} \in\left(y_{n} h, y_{n+1} h\right)$ such that

$$
\left|\sqrt{\lambda_{n+1}}-\sqrt{\lambda_{n}}\right|=2\left|\cos \left(y_{n+1} h\right)-\cos \left(y_{n} h\right)\right|=2 h\left|\sin \xi_{n}\right|\left|y_{n+1}-y_{n}\right| .
$$

Let $n_{T}^{\prime}=2\left[\frac{1}{T}\right]+2$. Since $y_{n}>\pi n$ for each $\varrho \ln N \leqslant n \leqslant N$ and $y_{N-n_{T}^{\prime}}<\left(N-n_{T}^{\prime}+\frac{1}{2}\right) \pi$, we deduce that $\pi \varrho h \ln N<\xi_{n}<\pi-\pi h\left(n_{T}^{\prime}+\frac{1}{2}\right)$ and, consequently,

$$
\left|\sin \xi_{n}\right| \geqslant h\left(2 n_{T}^{\prime}+1\right) \quad\left(\varrho \ln N \leqslant n \leqslant N-n_{T}^{\prime}\right) .
$$

By taking into account the last inequality, (3.5) and (3.6), we deduce immediately that

$$
\left|\sqrt{\lambda_{n+1}}-\sqrt{\lambda_{n}}\right| \geqslant \frac{\pi}{2} h^{2}\left(2 n_{T}^{\prime}+1\right) \geqslant \frac{2 \pi}{T} h^{2} \quad\left(\varrho \ln (N) \leqslant n \leqslant N-n_{T}^{\prime}\right) .
$$

On the other hand, by using Corollary 2.20 (with $\varepsilon=1$ ) and choosing $n_{T}^{\prime \prime}=\left[\frac{2 \pi}{T d}\right]+1$, we deduce that

$$
\left|\sqrt{\lambda_{n+1}}-\sqrt{\lambda_{n}}\right| \geqslant \frac{2 \pi}{T} h^{2} \quad\left(n_{T}^{\prime \prime} \leqslant n \leqslant N^{\frac{1}{6}}\right) .
$$

From (3.7) and (3.8) we deduce that (3.2) holds with $n_{T}=\max \left\{n_{T}^{\prime}, n_{T}^{\prime \prime}\right\}$.

Finally, we have all the ingredients needed to prove the main result of this paper.

Theorem 3.2. Let $T>0$ and $\gamma \in(0,1)$. There exists $N_{0} \in \mathbb{N}$ such that for every $N \geqslant N_{0}$ the observability inequality (1.11) holds with a positive constant $K_{T}$ independent of $h$ for every solution of (1.12) with initial data in the space $C_{h}(\gamma)$ given by (3.1). Moreover,

$$
\lim _{h \rightarrow \infty} \sup \left\{\frac{\left\|Y_{h}^{0}\right\|_{2}^{2}+\left\|Y_{h}^{1}\right\|_{0}^{2}}{\int_{0}^{T}\left|\frac{Y_{h N}(t)}{h^{2}}\right|^{2} d t} \mid\left(\begin{array}{c}
Y_{h}^{0} \\
Y_{h}^{1}
\end{array}\right) \in \mathbb{C}^{2 N} \text { and }\left(\begin{array}{c}
Y_{h} \\
\dot{Y}_{h}
\end{array}\right) \text { solution of }(1.12)\right\}=\infty .
$$

Proof. If the initial data $\left(\begin{array}{c}Y_{h}^{0} \\ Y_{h}^{1}\end{array}\right) \in \mathbb{C}^{2 N}$ is given by $\left(\begin{array}{c}Y_{h}^{0} \\ Y_{h}^{1}\end{array}\right)=\sum_{1 \leqslant|n| \leqslant \gamma N} a_{n} \Phi^{n}$, then the corresponding solution of (1.12) can be written as

$$
\left(\begin{array}{c}
Y_{h}(t) \\
\dot{Y}_{h}(t)
\end{array}\right)=\sum_{1 \leqslant|n| \leqslant \gamma N} a_{n} e^{-i \operatorname{sgn}(n) \frac{\sqrt{\lambda_{|n|}}}{h^{2}} t} \Phi^{n}
$$

Consequently, inequality (1.11) is equivalent to

$$
\sum_{1 \leqslant|n| \leqslant \gamma N}\left|a_{n}\right|^{2} \leqslant K_{T} \int_{0}^{T}\left|\sum_{1 \leqslant|n| \leqslant \gamma N} a_{n} e^{-i \operatorname{sgn}(n) \frac{\sqrt{\lambda_{|n|}}}{h^{2}} t} \frac{\phi_{N}^{|n|}}{\sqrt{\lambda_{|n|}}}\right|^{2} d t \quad\left(\left(a_{n}\right)_{1 \leqslant|n| \leqslant N} \in \mathbb{C}^{2 N}\right) .
$$

Now, by taking into account property (3.2) of the eigenvalues and using the generalization of Ingham's inequality proved in [10, Lemma 2.3], we obtain that there exist a constant $K_{T}^{\prime}>0$ and $N_{0} \in \mathbb{N}^{*}$, independent of $h$, such that the following inequality is verified for any $N \geqslant N_{0}$ and $\left(a_{n}\right)_{1 \leqslant|n| \leqslant N} \in \mathbb{C}^{2 N}$,

$$
\sum_{1 \leqslant|n| \leqslant \gamma N}\left|a_{n}\right|^{2}\left|\frac{\phi_{N}^{|n|}}{\sqrt{\lambda_{|n|}}}\right|^{2} \leqslant K_{T}^{\prime} \int_{0}^{T}\left|\sum_{1 \leqslant|n| \leqslant \gamma N} a_{n} e^{-i \operatorname{sgn}(n) \frac{\sqrt{\lambda_{|n|}}}{h^{2}}} t \frac{\phi_{N}^{|n|}}{\sqrt{\lambda_{|n|}}}\right|^{2} d t .
$$

On the other hand, let us remark that, if $n \leqslant \gamma N$, then

$$
\lambda_{n}=16 \sin ^{4}\left(\frac{y_{n} h}{2}\right) \leqslant 16 \sin ^{4}\left(\frac{(n+1) h \pi}{2}\right) \leqslant 16 \sin ^{4}\left(\frac{(\gamma+h) \pi}{2}\right)
$$


and there exists $\sigma \in(0,1)$ such that $\lambda_{n} \leqslant 16-\sigma$. According to Theorem 2.24 the following estimate holds for the last component of the eigenvectors $\phi^{n}$ of the matrix $A$

$$
\left|\phi_{N}^{n}\right| \geqslant K^{\prime \prime} \sqrt{\lambda_{n}} \quad(1 \leqslant n \leqslant \gamma N),
$$

where $K^{\prime \prime}$ is a positive constant depending on $\gamma$ but which is independent of $h$. From (3.11) and (3.12) it follows that (3.10) holds with $K_{T}=\frac{K_{T}^{\prime}}{K^{\prime \prime}}$.

In order to show (3.9), it is sufficient to consider $\left(\begin{array}{c}Y_{h}^{0} \\ Y_{h}^{1}\end{array}\right)=\Phi^{N}$ and to remark that, according to estimate (2.69) from Theorem 2.16,

$$
\frac{\left\|Y_{h}^{0}\right\|_{2}^{2}+\left\|Y_{h}^{1}\right\|_{0}^{2}}{\int_{0}^{T}\left|\frac{Y_{h N}(t)}{h^{2}}\right|^{2} d t}=\frac{\sqrt{\lambda_{N}}}{\left|\phi_{N}^{N}\right|}=O\left(\frac{1}{h}\right)
$$

and the proof of the theorem is complete.

Remark 3.3. Theorem 3.2 shows that a uniform observability inequality for the solutions of (1.12) holds true if the eigenfrequencies $\left|\lambda_{n}\right|>C / h^{4}$ are filtered out. Moreover, it shows that there are solutions containing eigenfrequencies in this range which are nor uniformly observable. These results are similar to the ones obtained for the hinged beam equation in [10]. These observability results allow to show immediately that we can control, with uniformly bounded controls, the projection over the space $\mathcal{C}_{h}(\gamma)$ of any solution of (1.5).

Remark 3.4. The observability result given by Theorem 3.2 cannot be obtained by using only the error estimates for the numerical scheme. Indeed, (2.73) would allow us to prove that the uniform observability holds for a space of initial data in which the eigenfrequencies $\left|\lambda_{n}\right| \geqslant \frac{C}{h^{\frac{4}{3}-\varepsilon}}$ are filtered out. This weaker result is similar to the ones obtained for more general hyperbolic problems in [3, 4]. Theorem 3.2, based on the asymptotic analysis of the highes eigenfrequencies, allows to filter at the range of the largest eigenvalue, which may improve the convergence rate of the numerical scheme.

\section{Numerical examples}

In this section we discuss some examples concerning the numerical approximation of controls associated to an initial data in $L^{2}(0,1) \times H^{-2}(0,1)$. More precisely, we consider the following initial data

$$
u_{0}(x)=\mathbb{1}_{\left(\frac{1}{4}, \frac{3}{4}\right)}(x), \quad u_{1}(x)=0 \quad(x \in(0,1)) .
$$

The aim of this section is to illustrate the theoretical results proved in the previous sections, namely the uniform controllability of filtered solutions.

In order to filter the high frequencies, for a given value of $N$, we firstly numerically approach the eigenvectors $\left(\phi^{n}\right)_{1 \leq n \leq N}$ and their corresponding eigenvalues $\left(\lambda_{n}\right)_{1 \leq n \leq N}$ of the matrix $A$. For each value of $N$ we compute the control associated to initial data given by (4.1) and filtered at the level $[\gamma N]$, where $\gamma$ is a filtering threshold and is chosen in the set $\{0.1,0.5,0.9,1\}$. More precisely, we consider initial displacements of the form

$$
u_{0}^{\gamma}=\sum_{n=1}^{[\gamma N]}\left\langle u_{0}, \phi^{n}\right\rangle_{0} \phi^{n} \in \mathbb{C}^{N},
$$

while the initial velocity is taken equal to zero. Figure 3 (left) displays initial data $u_{0}$ given by (4.1) filtered for these values of $\gamma$.

Since filtered solutions of the semi-discrete beam equation (1.6) are expressed by the sum given in (3.1), in order to represent correctly these solutions we take the time discretization step $\Delta t=\frac{\sqrt{\lambda_{[\gamma N]}}}{h^{2}}$. With this notation $\lambda_{[\gamma N]}$ corresponds to the highest frequency present in the solution to control. For the time discretization we use a Newmark finite-differences scheme of parameters $\frac{1}{4}$ and $\frac{1}{2}$ (see, for instance, the monograph $[6])$. 
A standard conjugate gradient algorithm was employed in order to minimize a weighted dual functional associated to the controllability problem. In order to keep solutions in the class $\mathcal{C}_{h}(\gamma)$ at each iteration of the conjugate gradient algorithm we filter the frequencies higher than $\lambda_{\gamma N}$ which appear as a consequence of the non-homogeneous boundary terms. Table 1 gathers the number of iterations needed for the convergence of the conjugate gradient algorithm. As a stopping criteria, the residuum in the conjugate gradient algorithm should be smaller than $\epsilon=10^{-7}$. We observe that for a fixed value of $N$ the number of iterations necessary for the convergence of the conjugate gradient algorithm increases when $\gamma$ increases. Moreover, for $\gamma=1$ the number of iterations increases linearly with respect to the number $N$ of discretization points and for values of $\gamma$ smaller than 1 this number is clearly upper bounded. This is related to the non-uniform observability of the highest frequencies and to the uniform observability of the filtered solutions, respectively.

\begin{tabular}{||c|c|c|c|c||}
\hline & $\gamma=0.1$ & $\gamma=0.5$ & $\gamma=0.9$ & $\gamma=1$ \\
\hline$N=25$ & 4 & 6 & 12 & 29 \\
$N=50$ & 4 & 6 & 15 & 52 \\
$N=100$ & 4 & 6 & 17 & 87 \\
$N=200$ & 4 & 6 & 20 & 168 \\
$N=400$ & 4 & 6 & 19 & 321 \\
\hline
\end{tabular}

Table 1: Number of iterations needed for the convergence of the conjugate gradient algorithm for initial data $\left(u_{0}^{\gamma}, 0\right)$ and different values of $N$.

In Table 2 we list the $L^{2}$ norms of the computed controls associated to initial data $\left(u_{0}^{\gamma}, 0\right)$ for different values of $N$. The values of the final state of the solutions controlled by these controls are gathered in Table 3.
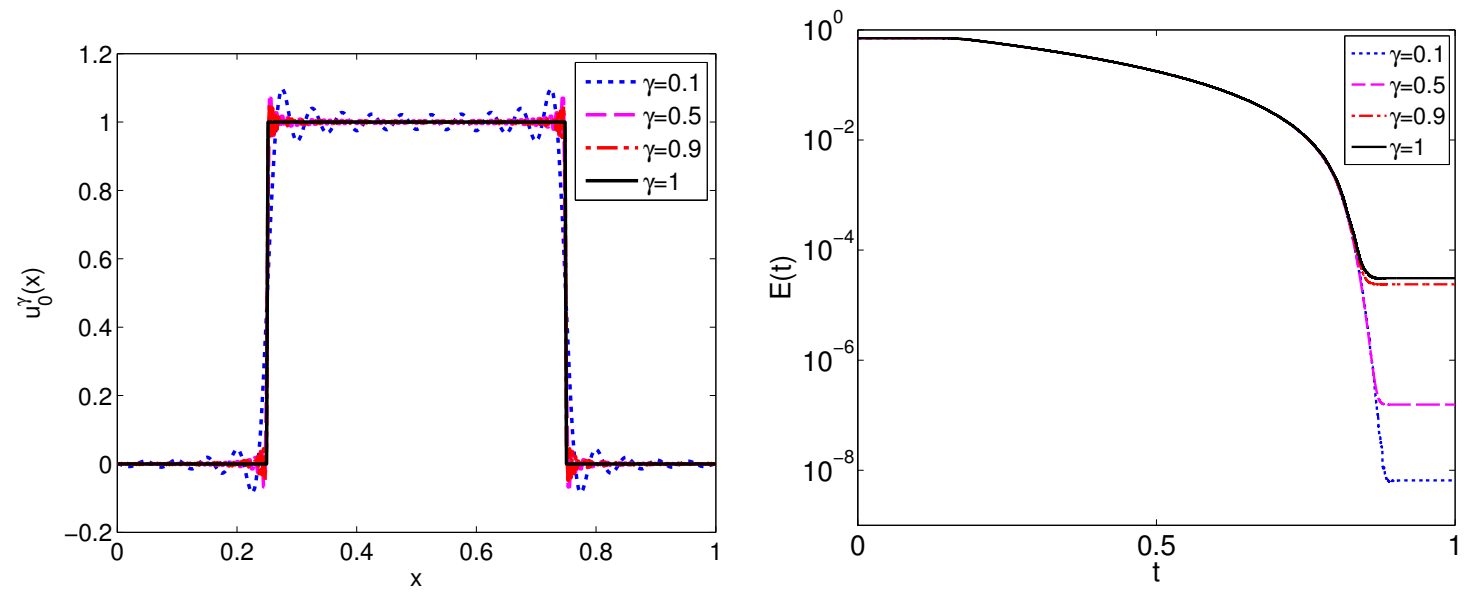

Figure 3: Filtered initial data $u_{0}^{\gamma}$ for different values of $\gamma$ and $N=400$ (left) and the energy of the corresponding controlled solutions (right).

\begin{tabular}{||c|c|c|c|c||}
\hline & $\gamma=0.1$ & $\gamma=0.5$ & $\gamma=0.9$ & $\gamma=1$ \\
\hline$N=25$ & 0.5691 & 0.5992 & 0.6411 & 0.9871 \\
$N=50$ & 0.5986 & 0.6082 & 0.6462 & 0.8104 \\
$N=100$ & 0.5880 & 0.5971 & 0.6104 & 0.8025 \\
$N=200$ & 0.5927 & 0.5982 & 0.6064 & 0.8031 \\
$N=400$ & 0.5960 & 0.5987 & 0.6028 & 0.8033 \\
\hline
\end{tabular}

Table 2: Norms $\left\|v_{h}\right\|_{L^{2}(0, T)}$ of controls associated to filtered solutions in $\mathcal{C}_{h}(\gamma)$ for different values of $N$ and $\gamma$.

Figure 4 displays controls associated to initial data $\left(u_{0}^{\gamma}, 0\right)$ for $\gamma \in\{0.1,0.5,0.9,1\}$ and $N=400$. In Figure 3 (right) we depict the evolution of the energy of solutions associated to these controls. As 


\begin{tabular}{||c|c|c|c|c||}
\hline & $\gamma=0.1$ & $\gamma=0.5$ & $\gamma=0.9$ & $\gamma=1$ \\
\hline$N=25$ & $8.12 \times 10^{-4}$ & $6.85 \times 10^{-9}$ & $5.98 \times 10^{-7}$ & $1.24 \times 10^{-10}$ \\
$N=50$ & $4.98 \times 10^{-6}$ & $2.33 \times 10^{-8}$ & $9.75 \times 10^{-6}$ & $9.02 \times 10^{-7}$ \\
$N=100$ & $3.61 \times 10^{-9}$ & $1.03 \times 10^{-7}$ & $6.51 \times 10^{-6}$ & $3.89 \times 10^{-6}$ \\
$N=200$ & $5.05 \times 10^{-9}$ & $1.41 \times 10^{-7}$ & $8.99 \times 10^{-6}$ & $1.75 \times 10^{-5}$ \\
$N=400$ & $6.53 \times 10^{-9}$ & $1.56 \times 10^{-7}$ & $2.38 \times 10^{-5}$ & $3.08 \times 10^{-5}$ \\
\hline
\end{tabular}

Table 3: Energy norm of the controlled filtered solution at the time $T=1$.

expected, controls present higher higher rate oscillations when $\gamma$ is close to 1 . Nevertheless, the energy of the corresponding solutions seems to go to zero when the time is approaching the final time $T$ taken in this section equal to 1 . In the same time, due to the fact that the numerical resolution of the beam equation for initial data containing higher frequencies is less precise, the norms of the controlled solutions are larger for larger values of the filtering threshold $\gamma$.

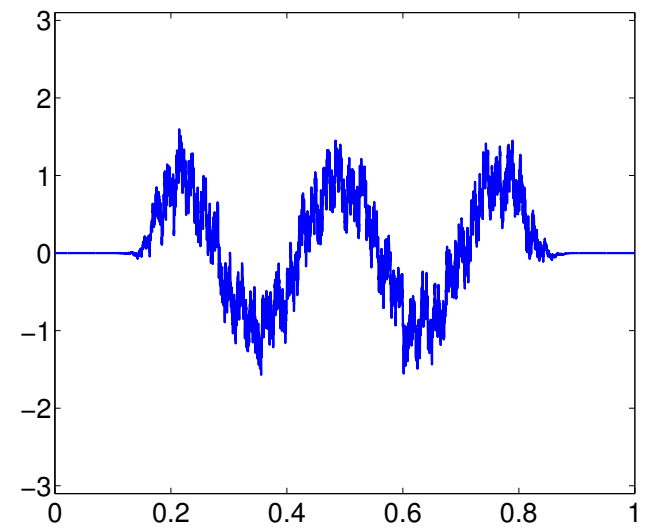

(a) $\gamma=0.1$

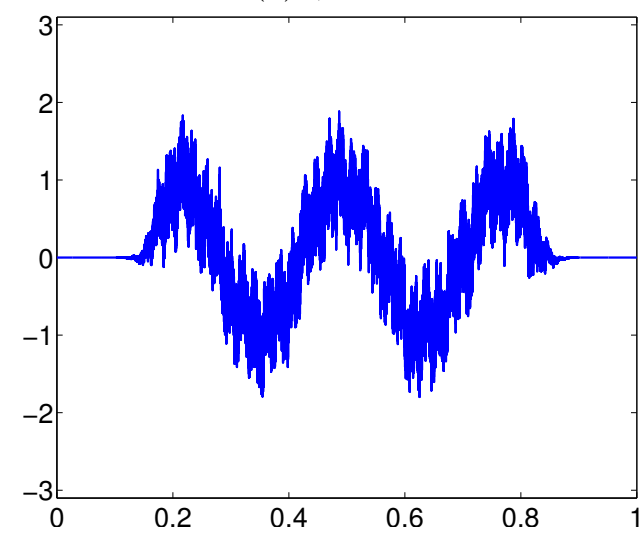

(c) $\gamma=0.9$

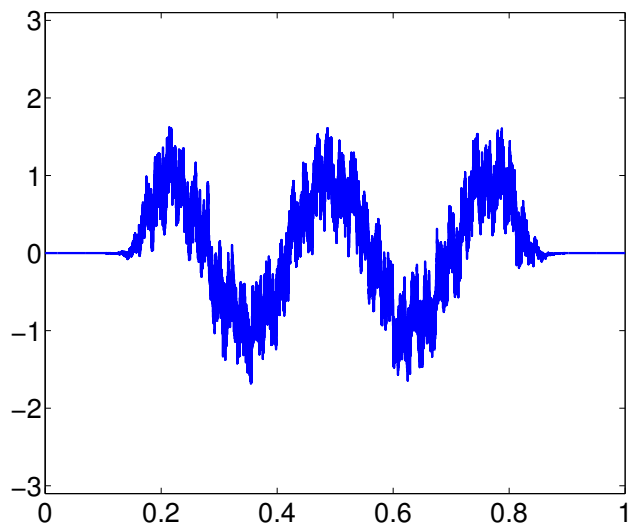

(b) $\gamma=0.5$

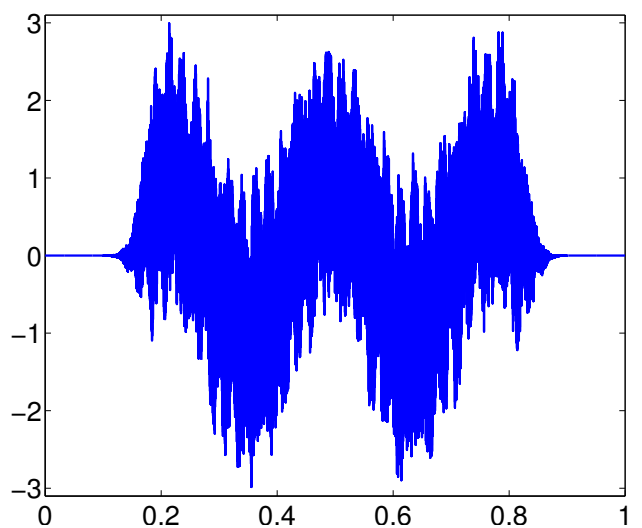

(d) $\gamma=1$

Figure 4: Controls obtained for $N=400$ and different values of the filtering threshold $\gamma$.

Finally, from a computational point of view, considering larger values of $N$ and unfiltered initial data could be difficult, since the time discretization step needed for a correct representation of such higher oscillating controls should be very small. Moreover, if no filtering strategy is employed $(\gamma=1)$ the number of iterations increases with $N$, this implying even longer computation times. 


\section{References}

[1] I. F. Bugariu, S. Micu, And I. RovenţA, Approximation of the controls for the beam equation with vanishing viscosity, Mathematics of Computation, published online: http://dx.doi.org/10.1090/mcom/3064 (2016).

[2] N. Cîndea, S. Micu, And I. RovenţA, Uniform observability for a finite differences discretization of a clamped beam equation, in Preprints of the 2nd IFAC Workshop on Control of Systems Governed by Partial Differential Equations, vol. accepted, 2016.

[3] S. ERvedozA, Spectral conditions for admissibility and observability of wave systems: applications to finite element schemes, Numer. Math., 113 (2009), pp. 377-415.

[4] _ Spectral conditions for admissibility and observability of Schrödinger systems: applications to finite element discretizations, Asymptot. Anal., 71 (2011), pp. 1-32.

[5] R. A. Horn and C. R. Johnson, Matrix analysis, Cambridge University Press, Cambridge, 1985.

[6] T. J. R. Hughes, The finite element method, Prentice Hall, Inc., Englewood Cliffs, NJ, 1987. Linear static and dynamic finite element analysis, With the collaboration of Robert M. Ferencz and Arthur M. Raefsky.

[7] J. A. Infante And E. Zuazua, Boundary observability for the space semi-discretizations of the 1-D wave equation, M2AN Math. Model. Numer. Anal., 33 (1999), pp. 407-438.

[8] H. B. KELlER, On the accuracy of finite difference approximations to the eigenvalues of differential and integral operators, Numer. Math., 7 (1965), pp. 412-419.

[9] V. Komornik, Exact controllability and stabilization, RAM: Research in Applied Mathematics, Masson, Paris; John Wiley \& Sons, Ltd., Chichester, 1994. The multiplier method.

[10] L. LEÓN AND E. ZuAZUA, Boundary controllability of the finite-difference space semi-discretizations of the beam equation, ESAIM Control Optim. Calc. Var., 8 (2002), pp. 827-862 (electronic). A tribute to J. L. Lions.

[11] M. TuCSNAK And G. Weiss, Observation and control for operator semigroups, Birkhäuser Advanced Texts: Basler Lehrbücher. [Birkhäuser Advanced Texts: Basel Textbooks], Birkhäuser Verlag, Basel, 2009. 